\title{
Rayleigh-Taylor Instability of Viscoelastic Drops at High Weber Numbers
}

\author{
D.D. Joseph*, G.S. Beavers*, T. Funada** \\ *University of Minnesota, Minneapolis, MN 55455 \\ ** Numazu College of Technology, Ooka 3600, \\ Numazu, Shizuoka, Japan 410-8501
}

\section{Abstract}

Movies of the breakup of viscous and viscoelastic drops in the high speed airstream behind a shock wave in a shock tube have been reported by Joseph, Belanger and Beavers [1999]. A Rayleigh-Taylor stability analysis for the initial breakup of a drop of Newtonian liquid was presented in that paper. The movies, which may be viewed at http://www.aem.umn.edu/ research/Aerodynamic_Breakup, show that for the conditions under which the experiments were carried out the drops were subjected to initial accelerations of orders $10^{4}$ to $10^{5}$ times the acceleration of gravity. In the Newtonian analysis of Joseph, Belanger and Beavers the most unstable Rayleigh-Taylor wave fits nearly perfectly with waves measured on enhanced images of drops from the movies, but the effects of viscosity cannot be neglected. Here we construct a Rayleigh-Taylor stability analysis for an Oldroyd B fluid using measured data for acceleration, density, viscosity and relaxation time $\lambda_{1}$. The most unstable wave is a sensitive function of the retardation time $\lambda_{2}$ which fits experiments when $\lambda_{2} / \lambda_{1}=\mathrm{O}\left(10^{-3}\right)$. The growth rates for the most unstable wave are much larger than for the comparable viscous drop, which agrees with the surprising fact that the breakup times for viscoelastic drops are shorter. We construct an approximate analysis of Rayleigh-Taylor instability based on viscoelastic potential flow which gives rise to nearly the same dispersion relation as the unapproximated analysis.

\section{Introduction}

This paper is an extension of the paper by Joseph, Belanger and Beavers [1999] (hereafter called JBB) on the breakup of a liquid drop suddenly exposed to a high-speed airstream behind a shock wave in a shock tube. In JBB we presented several series of photographs taken from high speed movies showing the breakup of various liquids in the flow behind Mach 2 and Mach 3 shock waves. We also presented a Rayleigh-Taylor stability analysis for drops of Newtonian liquids. We extend that work in this paper to include a Rayleigh-Taylor stability analysis for three viscoelastic drops for which measured data are given by JBB. The extensive literature relevant to drop breakup in a high speed airstream is reviewed in JBB and will not be repeated here. The recent and fairly extensive literature on atomization of Newtonian (not viscoelastic) liquids is well represented in the papers by Hsiang and Faeth (1992), Hwang, Liu and Rietz 
(1996), Faeth (1996) and Liu and Rietz (1997). These results, and earlier drop breakup studies such as Krzeczkowski (1980), Wierzba (1990), Kitscha and Kocamustafaogullari (1989), and Stone (1994), are restricted to relatively low Weber and Reynolds numbers. The highest Weber and Reynolds data for drop breakup was reported by Hsiang and Faeth (1992) who worked under conditions for which the Weber numbers ranged from 0.5 to 1000 with Reynolds numbers from 300-1600. The excellent paper on Rayleigh-Taylor instability in elastic liquids by Aitken and Wilson [1993] is directly relevant to our work here and was not reviewed by JBB.

Aitken and Wilson [1993] studied the problem of the stability of an incompressible elastic fluid above a free surface to small disturbances. They derived dispersion relations for an Oldroyd fluid in the case where the fluid is bounded below by a rigid surface. When the retardation time and inertia are neglected the analysis predicts an unbounded growth rate at a certain Weissenberg number. The addition of inertia or retardation smoothes this singularity. The work presented here differs from that of Aitken and Wilson in the following ways; in our work the two fluids are unbounded; we construct both an exact analysis and an approximate analysis based on potential flow; we aim to apply the analysis of Rayleigh-Taylor instability of viscoelastic drops using measured data; we compute and present dispersion relations emphasizing the role of the most dangerous wave associated with the maximum growth rate and thereby emphasizing the role of the huge acceleration in the drop breakup problem due to Rayleigh-Taylor instability; and we use the maximum growth rate to define a breakup time.

Only a few studies of the breakup of viscoelastic drops have been published; Lane [1951], Wilcox, June, Braun and Kelly [1961], Matta and Tytus [1982], and Matta, Tytus and Harris [1983]. Matta and co-workers did studies at Mach numbers near one and less. They showed that threads and ligaments of liquid arise immediately after breakup, rather than the droplets which are seen in Newtonian liquids. We have verified these general observations for three different viscoelastic liquids ( $2 \%$ aqueous solution of polyox; $2 \%$ aqueous solution of polyacrylamide; $2.6 \%$ solution of polystyrene butylacrylate in tributyl phosphate) in high speed air behind shocks with shock Mach numbers as high as 3. Breakup sequences for these three liquids are presented in figures in JBB, which show just a few frames from the respective movies on our web page. The figures for polyox and polyacrylamide are reproduced here as figures 1-3. For comparison we also reproduce here as figure 4 the breakup of $1 \mathrm{~kg} / \mathrm{m} . \mathrm{sec}$ silicone oil whose viscosity closely matches that of the polyacrylamide solution $(0.96 \mathrm{~kg} / \mathrm{m} . \mathrm{sec})$. 


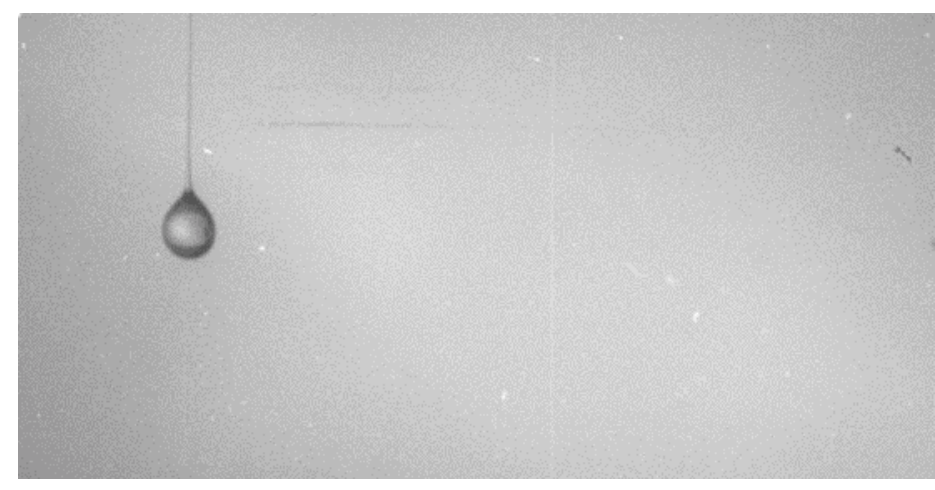

(a)

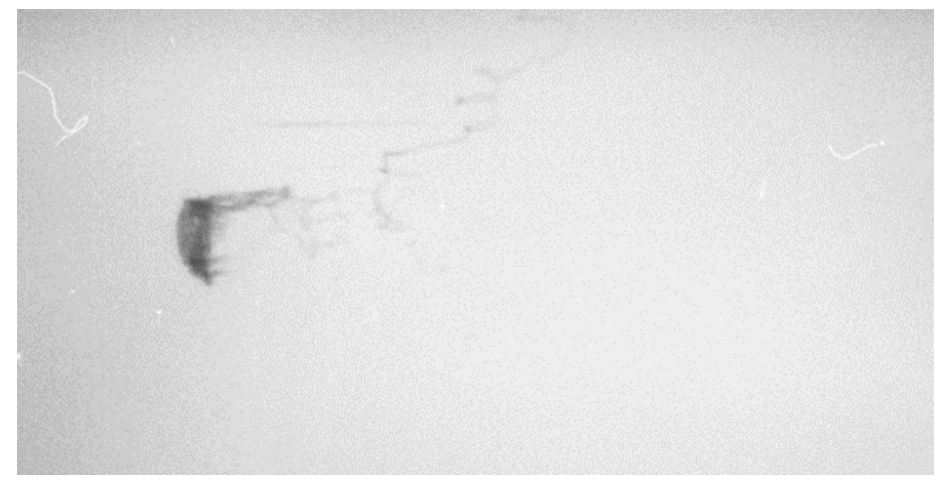

(c)

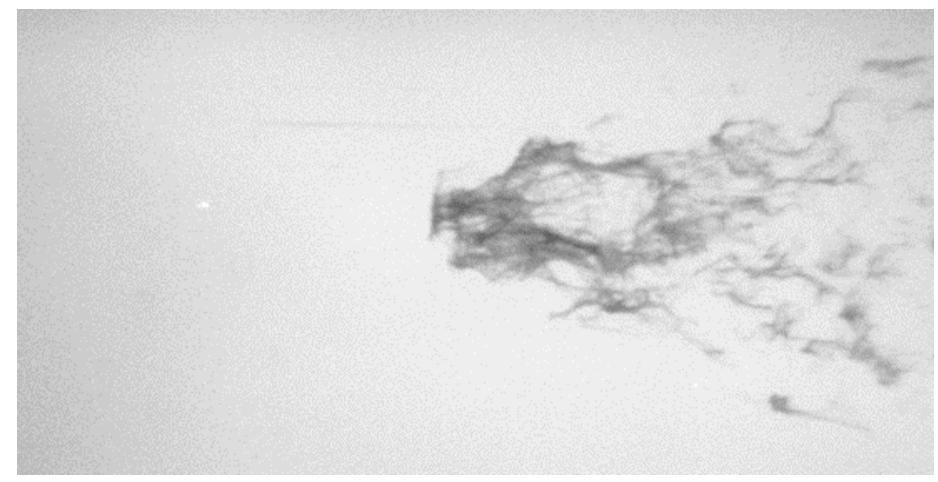

(e)

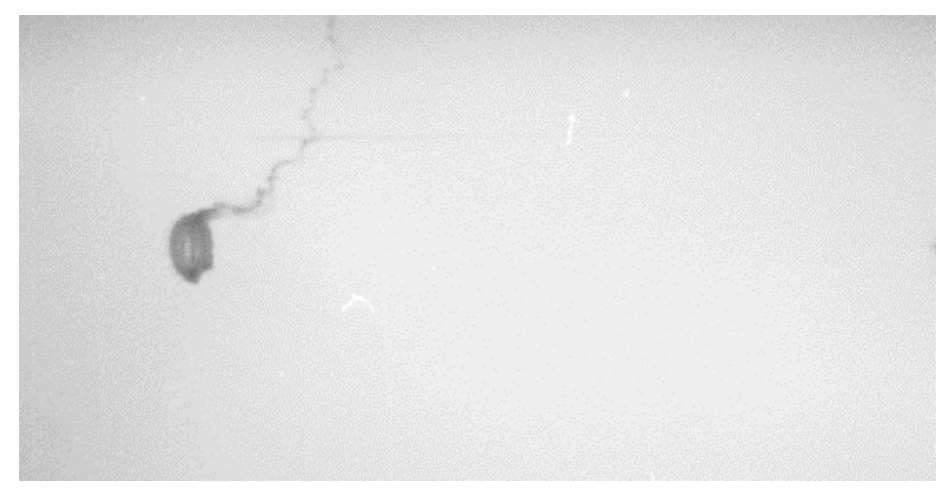

(b)

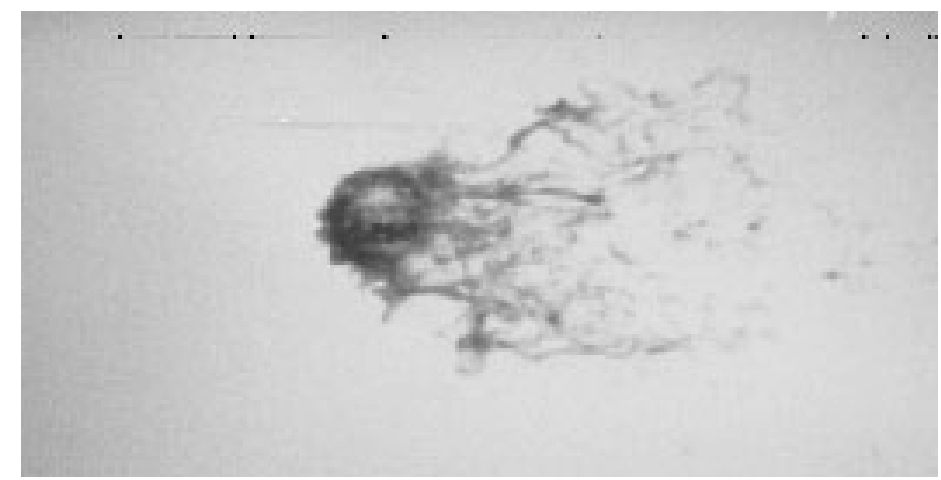

(d)

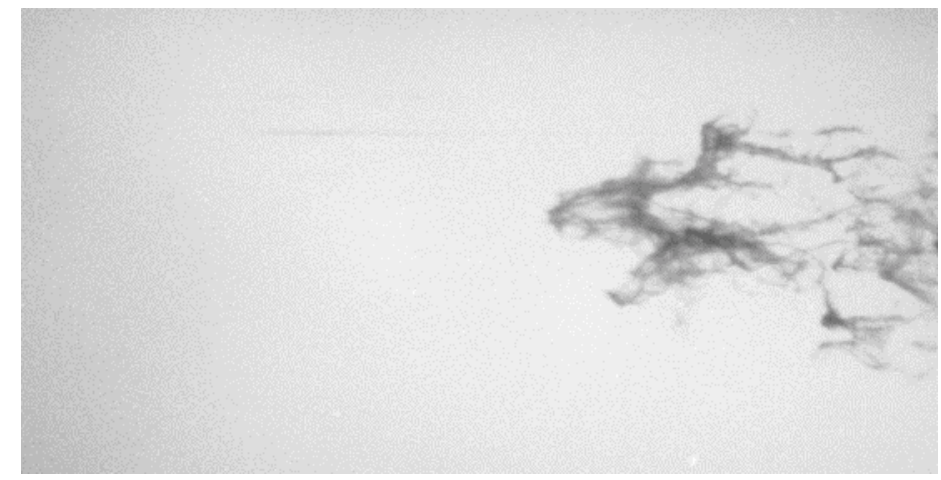

(f)

Figure 1. Stages in the breakup of a drop of 2\% aqueous solution of polyox (WSR 301; diameter $=2.9 \mathrm{~mm}$ ) in the flow behind a Mach 2 shock wave. Air velocity $=432 \mathrm{~m} / \mathrm{sec}$; dynamic pressure $=165.5 \mathrm{kPa}$; Weber no. $=15,200$. Time (microseconds): (a) 0 (b) 55 (c) 95 (d) 290 (e) 370 (f) 435 


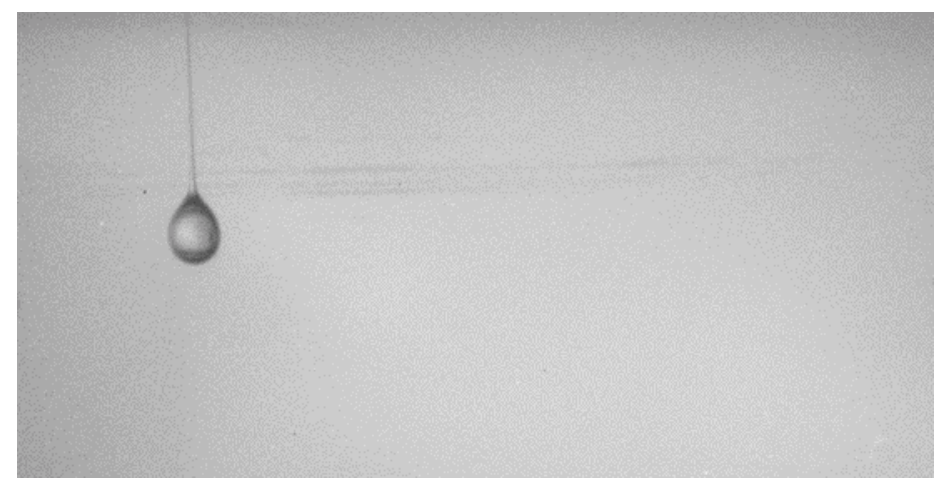

(a)

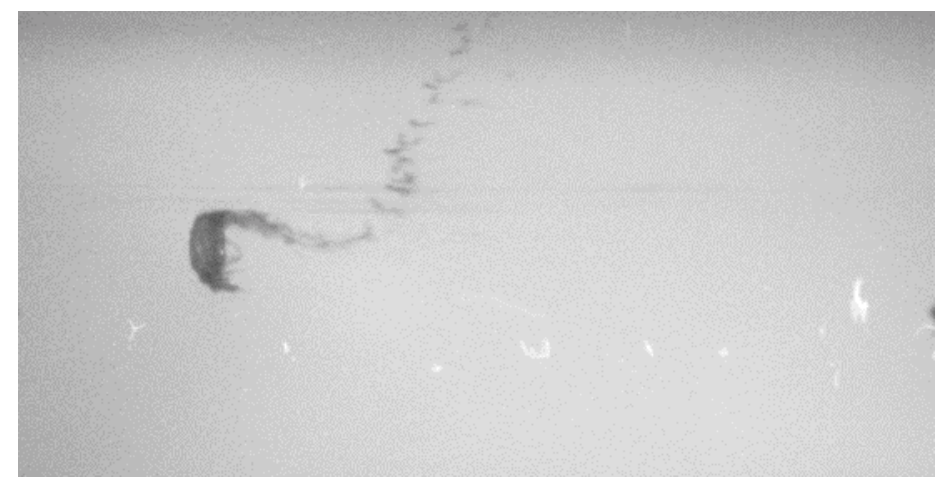

(c)

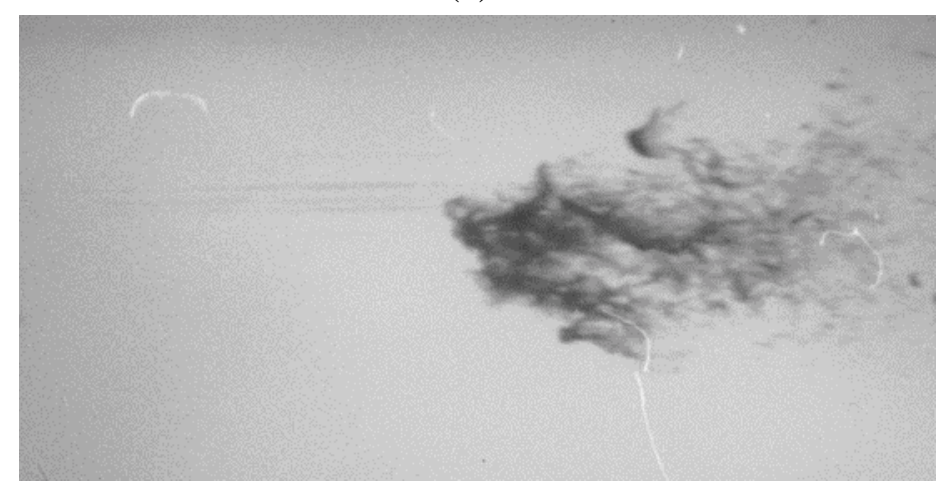

(e)

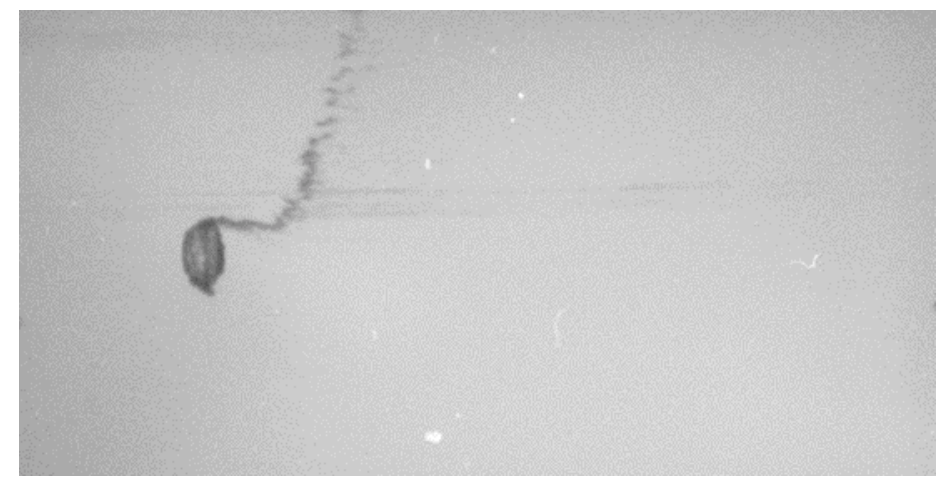

(b)

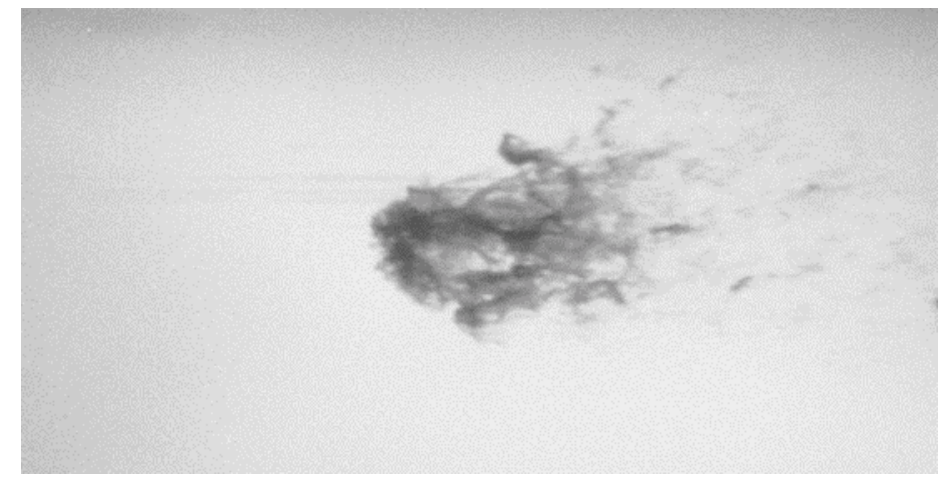

(d)

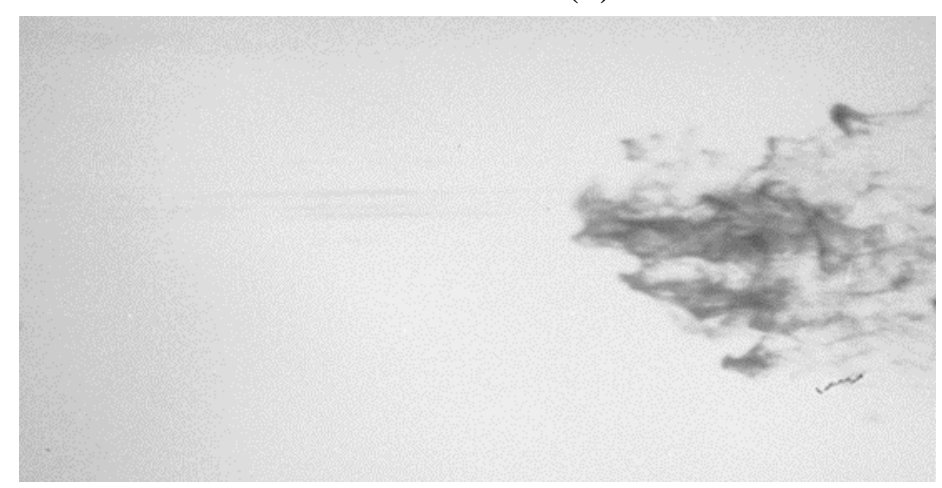

(f)

Figure 2. Stages in the breakup of a drop of $2 \%$ aqueous solution of polyox (WSR 301; diameter $=2.9 \mathrm{~mm}$ ) in the flow behind a Mach 3 shock wave. Air velocity $=755 \mathrm{~m} / \mathrm{sec}$; dynamic pressure $=587.2 \mathrm{kPa}$; Weber no. $=54,100$. Time (microseconds): (a) 0 (b) 30 (c) 45 (d) 170 (e) 195 (f) 235 
(a)

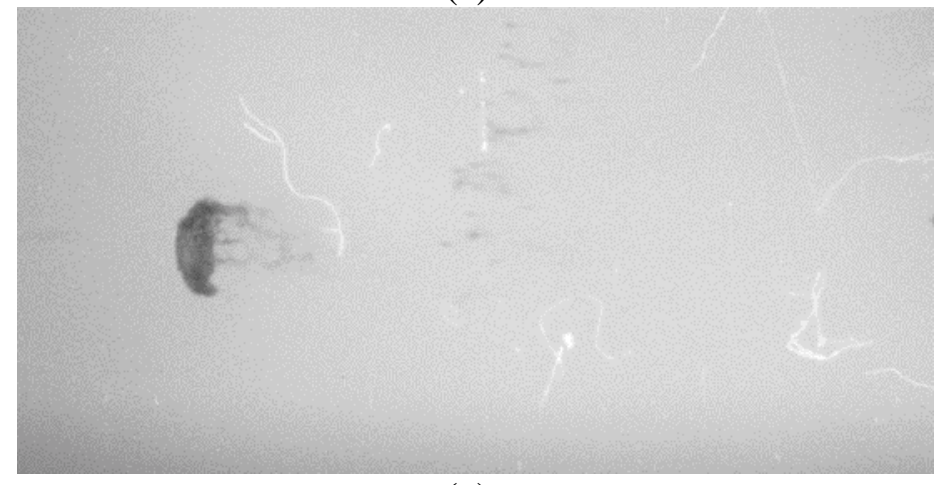

(c)

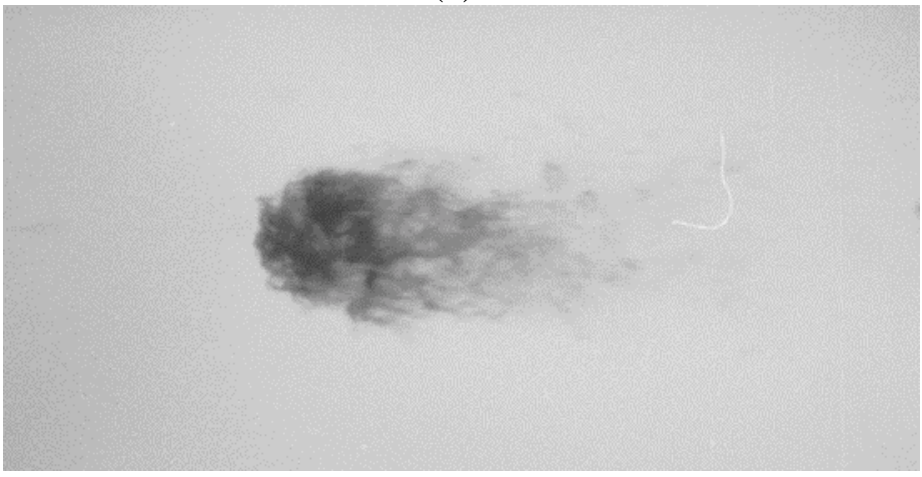

(e)

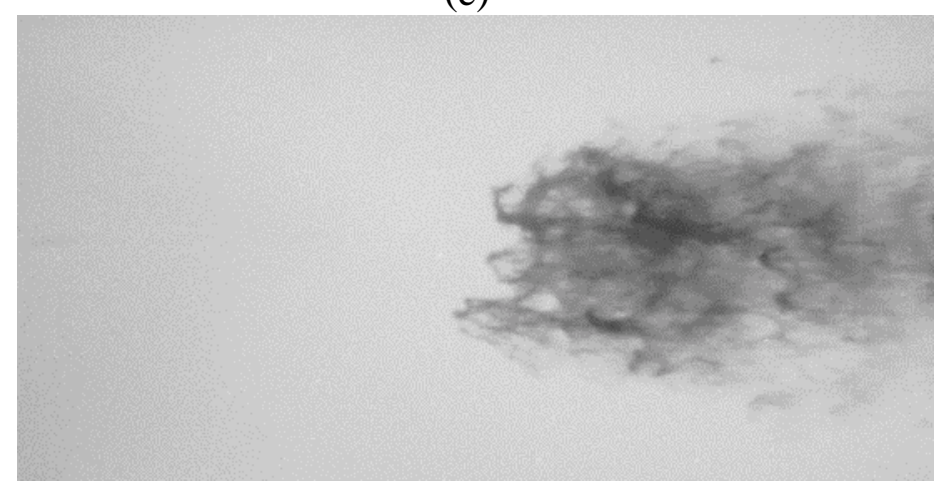

(g) (b)

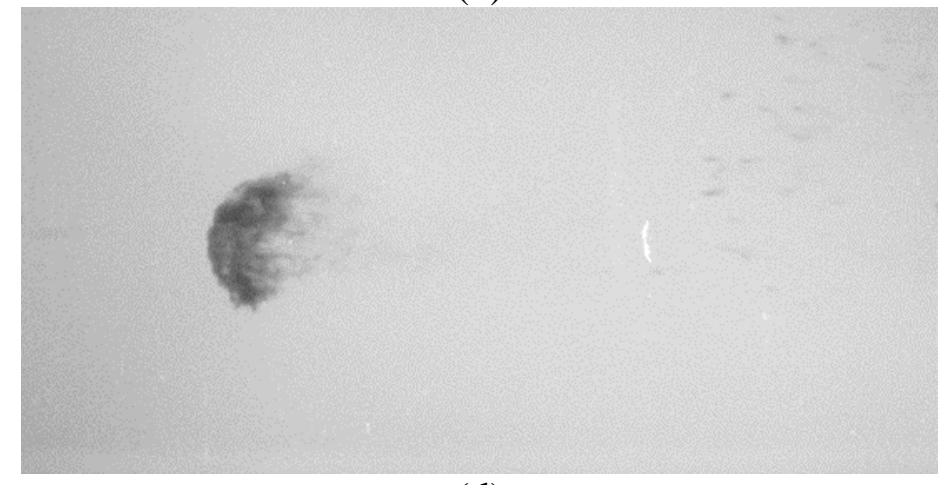

(d)

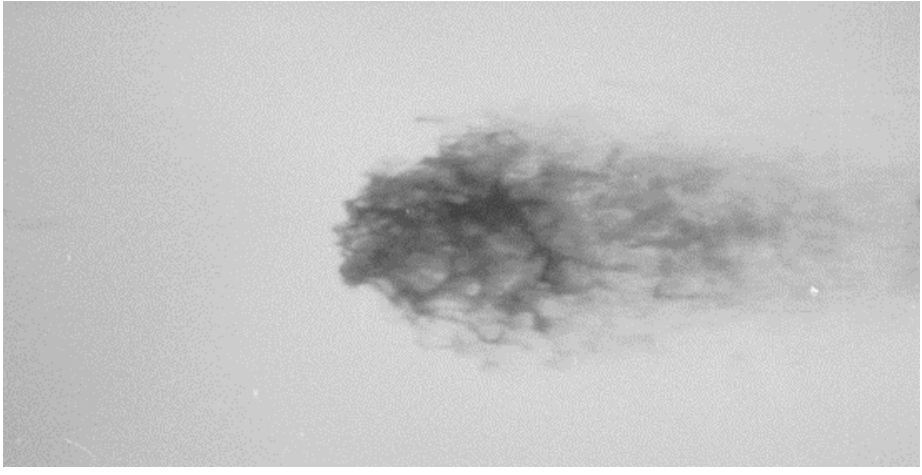

(f)

Figure 3. Stages in the breakup of a drop of $2 \%$ aqueous solution of polyacrylamide (Cyanamer N-300LMW; diameter $=3.2 \mathrm{~mm}$ ) in the flow behind a Mach 3 shock wave. Air velocity $=771 \mathrm{~m} / \mathrm{sec}$; dynamic pressure $=578.1 \mathrm{kPa}$; Weber no. $=82,200$.

Time (microseconds): (a) 0 (b) 45 (c) 60 (d) 90 (e) 145 (f) 185 (g) 225 


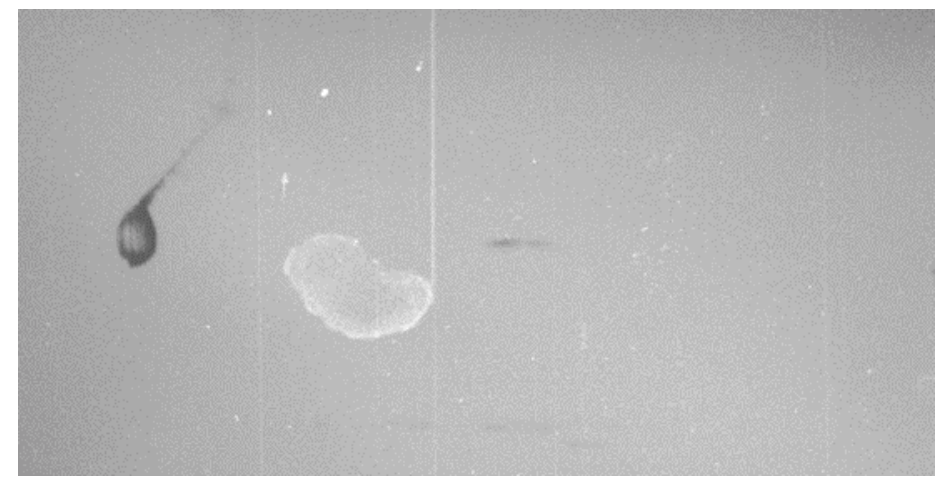

(a)

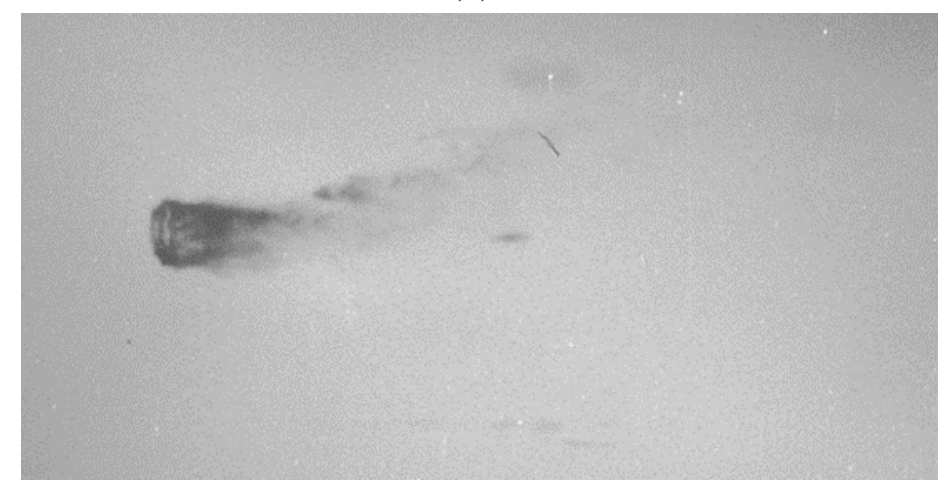

(c)

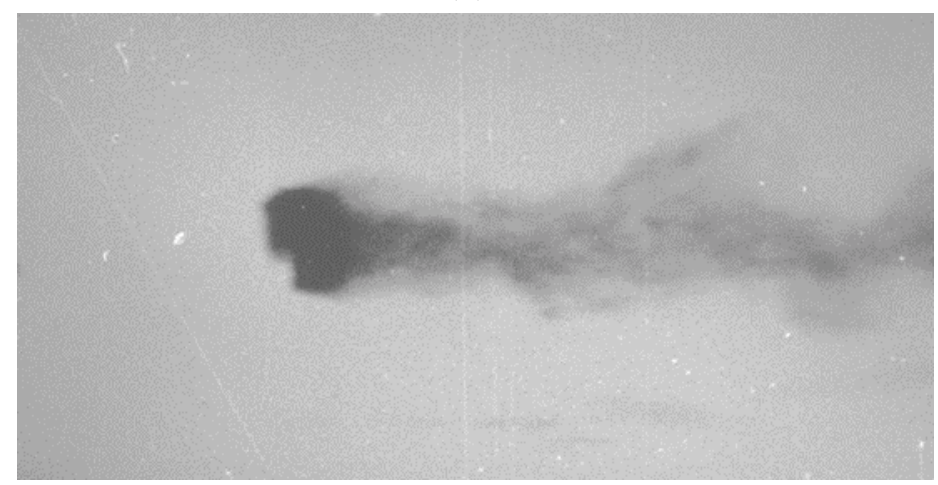

(e)

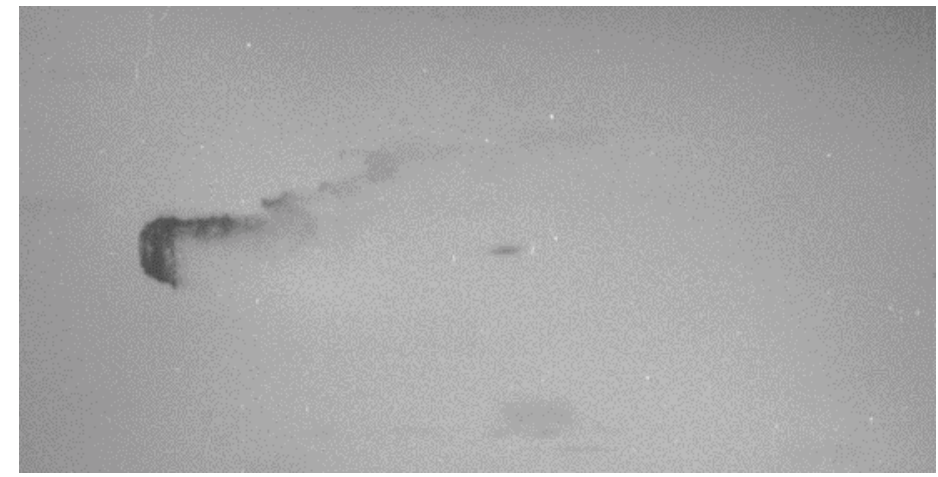

(b)

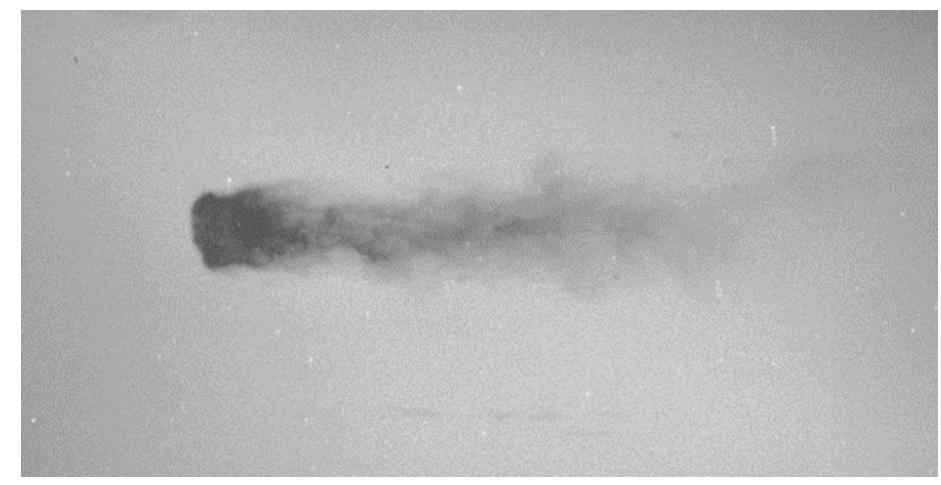

(d)

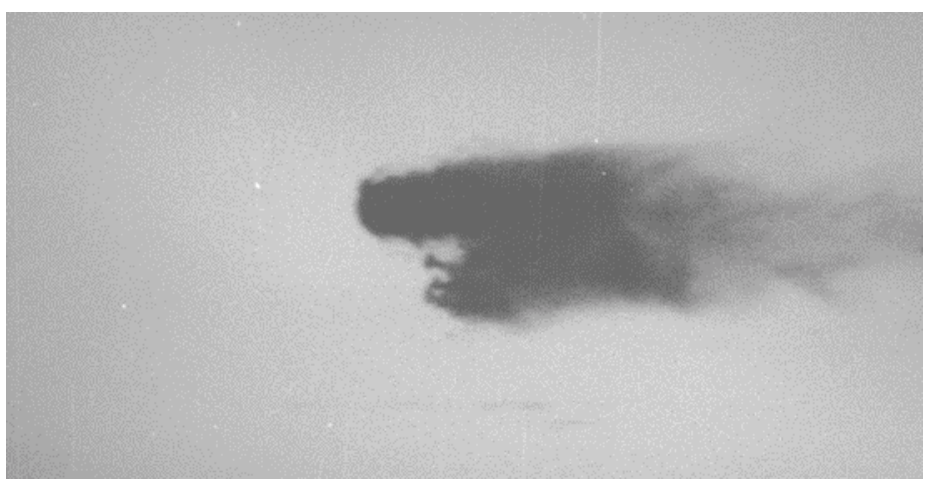

(f)

Figure 4. Stripping breakup of a drop of $1 \mathrm{~kg} / \mathrm{m} . \mathrm{sec}$ silicone oil (diameter $=2.6 \mathrm{~mm}$ ) in the flow behind a Mach 3 shock wave. Air velocity $=767 \mathrm{~m} / \mathrm{sec} ;$ dynamic pressure $=681.0 \mathrm{kPa}$ Weber no. $=168,600$.

Time (microseconds): (a) 15 (b) 40 (c) 50 (d) 80 (e) 115 (f) 150 


\section{Experiments}

The experiments reported here are fully described in JBB. The drops were injected into the test section of a shock tube and timed so that the shock wave passed over the drop as it fell under gravity into the field of view of a Cordin model 377 rotating drum camera, operated at 200,000 frames per second. The individual frames from the filmstrips produced by the camera were scanned into a PC as TIFF files using Adobe Photoshop and then composed into a movie sequence using Alias Composer running on a Unix-based workstation.

The data for the experiments discussed in this paper are listed in table 1. The Ohnesorge number $O h$, the Weber number We and the Reynolds number $R e$

$$
O h=\frac{\mu_{d}}{\left(\rho_{d} D \gamma\right)^{1 / 2}}, W e=\frac{\rho V^{2} D}{\gamma}, \quad R e=\frac{V D \rho}{\mu}
$$

are defined in terms of the initial drop diameter $D$, listed in the second column, drop viscosity $\mu_{d}$, surface tension $\gamma$, drop density $\rho_{d}$ listed in columns 3,4, and 5 and the free stream values of the velocity $V$, viscosity $\mu$, and density $\rho$.

\begin{tabular}{|r|c|c|c|c|c|c}
\hline & \multicolumn{7}{|c}{ LIQUID PROPERTIES } \\
\hline Liquid & $\begin{array}{c}\text { Diameter } \\
(\mathbf{m m})\end{array}$ & $\begin{array}{c}\text { Viscosity } \\
(\mathbf{k g} / \mathbf{m} . \mathbf{s e c})\end{array}$ & $\begin{array}{c}\text { Surface } \\
\text { Tension } \\
(\mathbf{N} / \mathbf{m})\end{array}$ & $\begin{array}{c}\text { Density } \\
\left(\mathbf{k g} / \mathbf{m}^{3}\right)\end{array}$ & $\begin{array}{c}\text { Relaxation } \\
\text { time }(\mathbf{s e c})\end{array}$ & Ohn. No. \\
\hline NEWTONIAN & & & & & & \\
SO 1000 & 2.6 & 1 & 0.021 & 969 & & 4.3 \\
SO 1000 & 2.6 & 1 & 0.021 & 969 & & 4.3 \\
VISCOELASTIC & & & & & & \\
2\% PO & 2.9 & 35 & 0.063 & 990 & 0.21 & 82.3 \\
$2 \%$ PO & 2.9 & 35 & 0.063 & 990 & 0.21 & 82.3 \\
$2 \%$ PAA & 3.2 & 0.96 & 0.045 & 990 & 0.039 & 2.5 \\
\hline
\end{tabular}

Table 1(a) 


\begin{tabular}{|c|c|c|c|c|c|c|c|c|}
\hline \multirow[b]{2}{*}{ Liquid } & \multicolumn{8}{|c|}{ FREE STREAM CONDITIONS } \\
\hline & $\begin{array}{c}\text { Velocity } \\
(\mathbf{m} / \mathbf{s})\end{array}$ & $\begin{array}{l}\text { Density } \\
\left(\mathrm{kg} / \mathrm{m}^{3}\right)\end{array}$ & $\begin{array}{c}\text { Pressure } \\
\text { (kPa) }\end{array}$ & $\begin{array}{c}\text { Dynamic } \\
\text { Pressure } \\
(\mathbf{k P a})\end{array}$ & $\begin{array}{c}\text { T2 Temp } \\
\text { (K) }\end{array}$ & $\begin{array}{c}\text { Weber No. } \\
\left(10^{3} x\right)\end{array}$ & $\begin{array}{c}\text { Reynolds } \\
\text { No. }\left(10^{3} x\right)\end{array}$ & Shock M \\
\hline \multicolumn{9}{|l|}{ NEWTONIAN } \\
\hline SO 1000 & 438.8 & 1.876 & 269.2 & 180.6 & 502 & 44.7 & 80.6 & 2.03 \\
\hline SO 1000 & 767.4 & 2.312 & 523.7 & 681.0 & 792 & 168.6 & 129.1 & 3.02 \\
\hline \multicolumn{9}{|l|}{ VISCOELASTIC } \\
\hline $2 \% \mathrm{PO}$ & 431.7 & 1.776 & 252.3 & 165.5 & 497 & 15.2 & 84.2 & 2.01 \\
\hline $2 \% \mathrm{PO}$ & 754.8 & 2.061 & 458.7 & 587.2 & 778 & 54.1 & 127.6 & 2.98 \\
\hline $2 \%$ PAA & 770.6 & 1.947 & 442.9 & 578.1 & 795 & 82.2 & 134.0 & 3.03 \\
\hline
\end{tabular}

Table 1(b). Experimental parameters. Relaxation time for $P O$ and $P A A$ are computed from measured values taken on the wave-speed meter, PO in the tables given by Joseph [1990] and PAA in the Ph.D Thesis of Y.J. Liu [1995].

\subsection{Displacement-time graphs and accelerations}

Displacement vs. time graphs for the Mach 3 experiments discussed in this paper are shown in figure 5. The Mach 2 graphs are of similar form. The distance refers to the slowest moving drop fragment (the windward stagnation point); other parts of the fragmenting drop accelerate

\begin{tabular}{|c|c|c|c|c|c|}
\hline Liquid & \multicolumn{2}{|c|}{ Silicone Oil } & \multicolumn{2}{|c|}{ 2\% Aqueous PO } & 2\% Aqueous PAA \\
\hline Viscosity (kg/m.sec) & \multicolumn{2}{|c|}{1} & \multicolumn{2}{|c|}{35} & 0.96 \\
\hline Shock Mach No. & 2 & 3 & 2 & 3 & 3 \\
\hline$\alpha\left(\mathrm{m} / \mathrm{sec}^{2}\right)$ & 1.463E5 & 5.561E5 & $0.687 \mathrm{E} 5$ & $3.240 \mathrm{E} 5$ & $2.461 \mathrm{E} 5$ \\
\hline $\mathbf{x}_{\mathbf{0}}(\mathbf{m})$ & $-28.5 E-5$ & $7.45 \mathrm{E}-5$ & $-17.7 E-5$ & $-0.046 \mathrm{E}-5$ & $-6.16 \mathrm{E}-5$ \\
\hline $\mathbf{t}_{\mathrm{o}}(\mathrm{sec})$ & $-3.43 E-5$ & $0.21 \mathrm{E}-5$ & $-5.07 \mathrm{E}-5$ & $-0.12 \mathrm{E}-5$ & $-1.49 \mathrm{E}-5$ \\
\hline $\begin{array}{r}\text { Initial Acceleration } \\
\left(\mathrm{m} / \mathrm{sec}^{2}\right)\end{array}$ & 2.92E5 & 11.12E5 & $1.37 \mathrm{E} 5$ & $6.48 \mathrm{E} 5$ & $4.92 \mathrm{E} 5$ \\
\hline $\begin{array}{r}\text { Max. Accel. From (3.2.8) } \\
\left((\mathrm{c}=\mathbf{0})\left(\mathrm{m} / \mathrm{sec}^{2}\right)\right.\end{array}$ & $1.07 \mathrm{E} 5$ & 4.05E5 & $0.86 \mathrm{E} 5$ & 3.07E5 & 2.74E5 \\
\hline $\begin{array}{r}\text { Mean Accel./Max. Accel. } \\
\text { From }(3.2 .8)\end{array}$ & 2.7 & 2.7 & 1.6 & 2.1 & 1.8 \\
\hline
\end{tabular}

Table 2. Curve-fitting parameters and initial accelerations for the liquid drops specified in table 1.

from rest even more rapidly. The graphs are nearly perfect parabolas for about the first two hundred microseconds of the motion, which allows the initial acceleration to be obtained by 
fitting a curve of the form $x-x_{o}=\alpha\left(t-t_{o}\right)^{2}$. Values of the parameters $\alpha, t_{o}, x_{o}$, and the initial acceleration are listed in table 2. It is noteworthy that in these graphs the acceleration is constant,
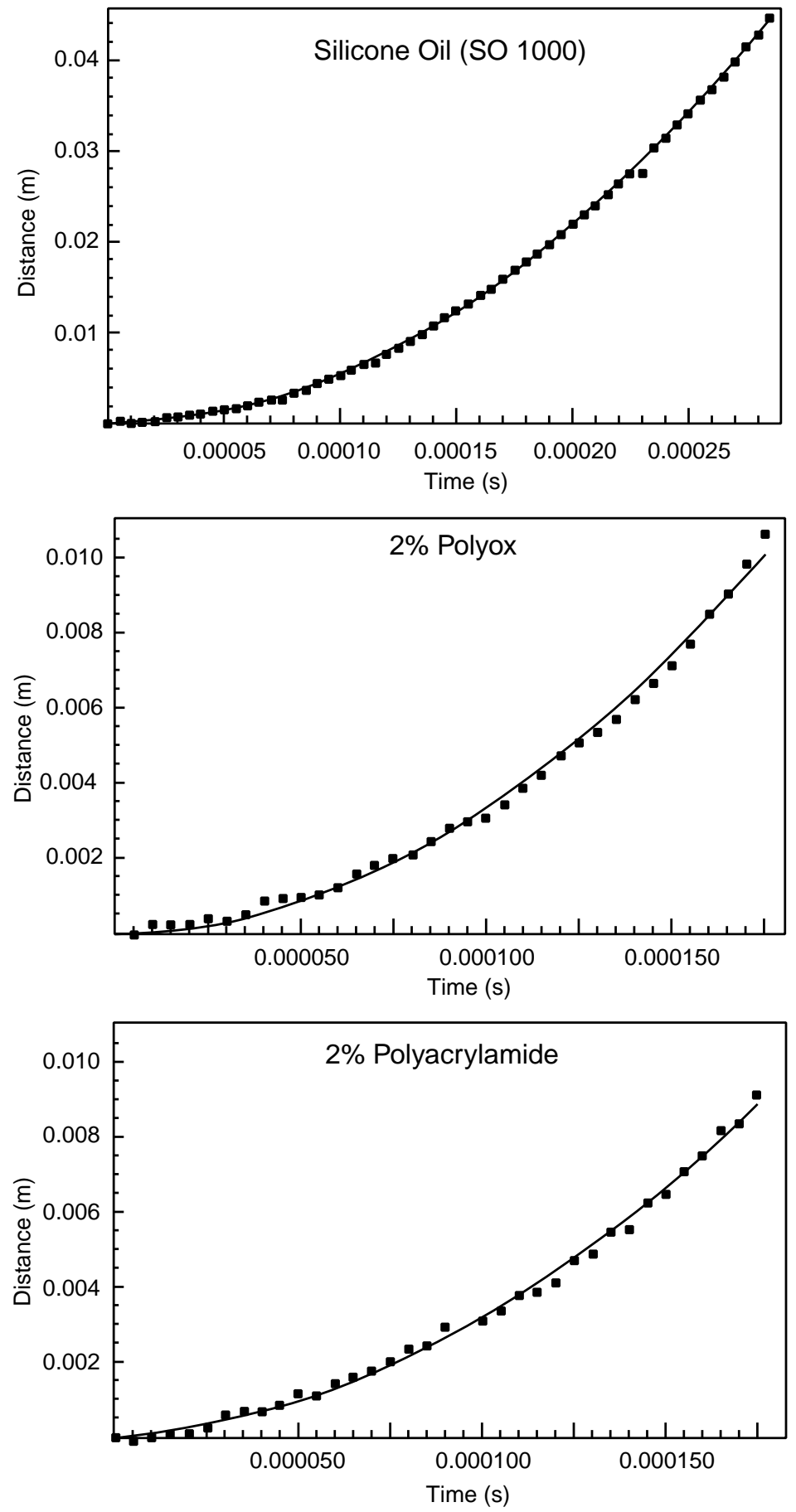

Distance traveled vs. time. $x-x_{0}=\alpha\left(t-t_{0}\right)^{2}$ where $x_{0}$ and $t_{0}$ are the extrapolated starting values from the curve fitting technique. The starting values $x_{0}$ and $t_{0}$ are uncertain within several pixels and several frames (5 $\mu$ sec per frame). 
independent of time for small times, and about $10^{4}-10^{5}$ times the acceleration of gravity depending upon the shock wave Mach number. In general there is a moderate drop-off of acceleration with time over the course of the several hundred microseconds that it takes to totally fragment the drop.

The initial accelerations are an increasing function of the shock Mach number; the dynamic pressure which accelerates the drop increases with the free stream velocity. At a fixed freestream dynamic pressure there appears to be a tendency for the acceleration to decrease with drop size. If we write that the drag on a spherical drop is proportional to the drop diameter squared and the mass to the diameter cubed, then the acceleration is proportional to $D^{-1}$ and decreases with increasing $D$.

\section{Theory}

\subsection{Background}

Rayleigh-Taylor instabilities (Taylor [1950]) always play a role in drop breakup. Rayleigh showed that a heavy fluid over a light fluid is unstable, as common experience dictates. $\mathrm{He}$ treated the stability of heavy fluid over light fluid without viscosity, and he found that a disturbance of the flat free surface grows exponentially like exp $(n t)$ where

$$
n={\frac{k g\left(\rho_{2}-\rho_{1}\right)}{\rho_{1}+\rho_{2}}}^{1 / 2}
$$

where $\rho_{2}$ is the density of the heavy fluid, $\rho_{1}$ is the density of the light fluid, $g$ is the acceleration of gravity and $k=2 \pi l$ is the wavenumber and is the wavelength. The instability described by (3.1.1) is catastrophic since the growth rate $n$ tends to infinity, at any fixed time, no matter how small, as the wavelength tends to zero. The solutions are unstable to short waves even at the earliest times. Such kinds of disastrous instabilities are called "Hadamard unstable" and the initial value problems associated with these instabilities are said to be "ill posed" (Joseph \& Saut [1990]). Ill-posed problems are disasters for numerical simulations. Because such problems are unstable to ever shorter waves, the finer the mesh, the worse the result.

Nature will not allow such a singular instability; for example, neglected effects like viscosity and surface tension will enter the physics strongly at the shortest wavelength. Surface tension eliminates the instability of the short waves; there is a finite wavelength depending strongly on viscosity as well as surface tension for which the growth rate $n$ is maximum. This is the 
wavelength that should occur in a real physical problem and would determine the wavelength on the corrugated fronts of breaking drops in a high speed air flow.

Taylor [1950] extended Rayleigh's inviscid analysis to the case where a constant acceleration of the superposed fluids other than gravity is taken into account. Assuming a constant value for the acceleration, Taylor [1950] showed that when two superposed fluids of different densities are accelerated in a direction perpendicular to their interface, this surface is unstable if the acceleration is directed from the lighter to the heavier fluid. The Taylor instability depends strongly on the value of the acceleration $a$; for example, if $g$ in (3.1.1) is replaced by $a=10^{4} \mathrm{~g}$, the growth rate $n$ is increased by a factor of $100.10^{4} \mathrm{~g}$ to $10^{5} \mathrm{~g}$ are representative values of the acceleration of drops in our shock tube; moreover the acceleration is nearly constant for all liquid drops at a given shock Mach number (see table 2 of JBB). A similar observation was made by Engel [1958]. Since the acceleration is perpendicular to the air-liquid interface and directed from gas to liquid, the accelerating liquid drop is unstable and is prey to the characteristic short wave corrugation associated with this instability.

The corrugations at the front of an unstable drop are driven toward the drop equator by shear flow of gas coming from the high pressure stagnation point. This shear flow may also be subject to an instability of the Kelvin-Helmholz type. Since the tangential velocity is zero at the stagnation point and small near the stagnation point, the Kelvin-Helmholz instability may not interact too strongly with the Rayleigh-Taylor instability.

\subsection{Acceleration}

The acceleration of the drop is a major factor in the Rayleigh-Taylor (RT) instability. It is instructive to see how the acceleration enters into the equations of motion. Suppose the lab frame is identified with $(\mathbf{X}, \hat{t})$ and the drop velocity is $\mathbf{v}(\mathbf{X}, \hat{t})$. Then we refer the equations of motion

$$
\rho\left(\frac{\partial \mathbf{v}}{\partial \hat{t}}+\mathbf{v} \bullet \frac{\partial \mathbf{v}}{\partial \mathbf{X}}\right)=\operatorname{div} \mathbf{T}+\rho \mathbf{g}, \frac{\partial}{\partial \mathbf{X}} \bullet \mathbf{v}=0
$$

to an accelerating frame in which the mass center of the drop is stationary identified with $(\mathbf{x}, t)$ and $\mathbf{v}(\mathbf{X}, \hat{t})=\mathbf{U}(\mathbf{x}, t)+\mathbf{V}(t), d \mathbf{X}=d \mathbf{x}+\mathbf{V}(t) d t, d \hat{t}=d t$. Then we find that

$$
\rho\left(\frac{\partial \mathbf{U}}{\partial t}+\mathbf{U} \bullet \frac{\partial \mathbf{U}}{\partial \mathbf{x}}\right)+\rho \dot{\mathbf{V}}=\operatorname{div} \mathbf{T}+\rho \mathbf{g}, \frac{\partial}{\partial \mathbf{x}} \bullet \mathbf{U}=0
$$

where $\mathbf{T}$ is the stress tensor.

The term

$$
\rho(\mathbf{g}-\dot{\mathbf{V}})
$$


enters into Rayleigh-Taylor instability and $\dot{\mathbf{V}}$ dominates in the drop breakup problem because the initial velocity is very small and the initial acceleration is very large.

The Rayleigh-Taylor instability occurs when $\dot{\mathbf{V}}$ is directed from the light to the heavy fluid as when the initially stationary drop is accelerated to the free stream velocity in the high speed airstream behind the shock in the shock tube or when a moving drop in a stagnant fluid is decelerated by the air to zero velocity. The analysis works well in air and liquid where the density and viscosity of air can be neglected with only small error; we get Rayleigh-Taylor instability in a vacuum because it is the drop acceleration term and not the material properties of air which induces the instability.

At early times the drop flattens under high pressure at the front and back of the drop; very soon thereafter the pressure recovery at the back of the drop falls due to the formation of a wake with low pressures like those associated with high speed flow at the side of the drop. At these early times we may estimate the terms in Newton's formula $F=m \dot{V}$. We approximate the drop shape as hemispherical with radius $R_{o}$ and volume $\frac{2}{3} \pi R_{o}^{3}$. The force is mainly due to the pressure drop front to back; at the front we have the dynamic pressure

$$
p_{f}=\rho U^{2} / 2
$$

whereas at the back

$$
p_{b}=c \rho U^{2} / 2
$$

with $c<1$. Then the force

$$
F=\left(p_{f}-p_{b}\right) \pi R_{o}^{2}=(1-c) \rho U^{2} \pi R_{o}^{2} / 2
$$

and

$$
m \dot{V}=\rho_{D} \frac{2}{3} \pi R_{o}^{3} \dot{V}
$$

where $\rho$ is the density of the air and $\rho_{D}$ the density of the liquid drops. Hence

$$
\dot{V}=\frac{3}{4}(1-c) \frac{\rho}{\rho_{D}} \frac{U^{2}}{R_{o}} .
$$

This formula predicts that the drop acceleration scales with the reciprocal of the drop radius as we mentioned before, and with the dynamic pressure. The acceleration $\dot{V}$ of the drop could be reduced to very low values in a low pressure environment even when the airspeed $U$ is large. 


\subsection{Stability Analysis}

The undisturbed interface between two fluids is located at $z=0$, with a system of Cartesian coordinates $\boldsymbol{x}=(x, y, z)=\left(x_{1}, x_{2}, x_{3}\right)$ moving with acceleration $\boldsymbol{a}$ :

$$
\boldsymbol{a}=\boldsymbol{g}-\dot{\boldsymbol{V}}=(0,-g,-\dot{V})=(0,-g,-a)
$$

For the conditions of the experiments described in this paper the drop moves in a horizontal plane and we may neglect $g$ as at least four orders of magnitude smaller than $\dot{V}$. The undisturbed rest state is given by the pressure $\bar{p}^{(2)}$ in the heavy non-Newtonian fluid (the Oldroyd B-fluid) in $z>0$ and $\bar{p}^{(1)}$ in the light Newtonian fluid in $z<0$ :

$$
\bar{p}^{(2)}=p_{0}-\rho_{2} a z, \quad \bar{p}^{(1)}=p_{0}-\rho_{1} a z
$$

where $p_{0}$ is the pressure at the interface, $\rho_{2}$ denotes the density of the heavy fluid, and $\rho_{1}$ is the density of the light fluid. Upon the undisturbed state, small disturbances are superimposed to give rise to the Rayleigh-Taylor instability, for which equations in the heavy fluid (in $0<z$ ) are given by

$$
\begin{gathered}
\rho_{2} \frac{\partial \boldsymbol{u}^{(2)}}{\partial t}=-\nabla p^{(2)}+\nabla \cdot \tau^{(2)}, \\
\nabla \cdot \boldsymbol{u}^{(2)}=0, \\
\tau_{i j}^{(2)}+\lambda_{1} \frac{\partial \tau_{i j}^{(2)}}{\partial t}=2 \mu_{2}\left(e_{i j}^{(2)}+\lambda_{2} \frac{\partial e_{i j}^{(2)}}{\partial t}\right), \\
e_{i j}^{(2)}=\frac{1}{2}\left(\frac{\partial u_{i}^{(2)}}{\partial x_{j}}+\frac{\partial u_{j}^{(2)}}{\partial x_{i}}\right),
\end{gathered}
$$

where $\boldsymbol{u}^{(2)}=\left(u^{(2)}, v^{(2)}, w^{(2)}\right)=\left(u_{1}^{(2)}, u_{2}^{(2)}, u_{3}^{(2)}\right)$ is the velocity disturbance, the viscous stress tensor $\tau_{i j}^{(2)}$ of the Oldroyd B-fluid is expressed as the constitutive equation (3.3.3c) with the strain tensor $e_{i j}^{(2)}$ and the viscosity $\mu_{2} ; \lambda_{1}$ is the relaxation time and $\lambda_{2}$ the retardation time; the conventional tensor notation is used here. Then, equations for disturbances in the light fluid (in $z<0$ ) are given by 


$$
\begin{gathered}
\rho_{1} \frac{\partial \boldsymbol{u}^{(1)}}{\partial t}=-\nabla p^{(1)}+\nabla \cdot \tau^{(1)} \\
\nabla \cdot \boldsymbol{u}^{(1)}=0 \\
\tau_{i j}^{(1)}=2 \mu_{1} e_{i j}^{(1)} \\
e_{i j}^{(1)}=\frac{1}{2}\left(\frac{\partial u_{i}^{(1)}}{\partial x_{j}}+\frac{\partial u_{j}^{(1)}}{\partial x_{i}}\right)
\end{gathered}
$$

where the viscous stress tensor $\tau_{i j}^{(1)}$ of the Newtonian fluid is expressed as (3.3.4c) with the strain tensor $e_{i j}^{(1)}$ and the viscosity $\mu_{1}$.

Boundary conditions at the interface with its displacement $h$ (at $z=h \approx 0$ ) are given by the continuity of velocity, the kinetic condition and the continuity of the stress:

$$
\begin{gathered}
\boldsymbol{u}^{(1)}=\boldsymbol{u}^{(2)}, \quad \frac{\partial h}{\partial t}=w^{(1)}=w^{(2)}, \\
\tau_{13}^{(1)}=\tau_{13}^{(2)}, \quad \tau_{23}^{(1)}=\tau_{23}^{(2)} \\
-p^{(2)}+\tau_{33}^{(2)}+\rho_{2} a h-\left(-p^{(1)}+\tau_{33}^{(1)}+\rho_{1} a h\right)=-\gamma \Delta h,
\end{gathered}
$$

where $\gamma$ is the surface tension and $\Delta$ is the horizontal Laplacian:

$$
\Delta=\frac{\partial^{2}}{\partial x^{2}}+\frac{\partial^{2}}{\partial y^{2}}
$$

Further, the boundary conditions require that the disturbances vanish, respectively, as $z \rightarrow \pm \infty$.

The solution to the system of the disturbances may take the following form:

$$
\left[\boldsymbol{u}^{(2)}, p^{(2)}, h, \boldsymbol{u}^{(1)}, p^{(1)}\right]=\left[\hat{\boldsymbol{u}}^{(2)}(z), \hat{p}^{(2)}(z), \hat{h}, \hat{\boldsymbol{u}}^{(1)}(z), \hat{p}^{(1)}(z)\right] \exp \left(n t+\imath k_{x} x+\imath k_{y} y\right)+c . c .
$$

where $n$ denotes the complex growth rate, $\left(k_{x}, k_{y}, 0\right)$ is the wavenumber vector of magnitude $k=$ $\sqrt{k_{x}^{2}+k_{y}^{2}}$, and c.c. stands for the complex conjugate of the preceding expression. Using (3.3.7), the constitutive equation $(3.3 .3 \mathrm{c})$ is now written as

$$
\tau_{i j}^{(2)}=2 \hat{\alpha} e_{i j}^{(2)}
$$

with $\hat{\alpha}$ defined by

$$
\hat{\alpha}=\mu_{2} \frac{1+\lambda_{2} n}{1+\lambda_{1} n} .
$$


Taking this into account and taking rotation of (3.3.3a) and (3.3.4a), using $\nabla \times \nabla \times \boldsymbol{u}=-\nabla^{2} \boldsymbol{u}$ for incompressible fluid, we get the following equations:

$$
\left(\nabla^{2}-\frac{n \rho_{1}}{\mu_{1}}\right) \nabla^{2} w^{(1)}=0 \quad \text { in } z<0, \quad\left(\nabla^{2}-\frac{n \rho_{2}}{\hat{\alpha}}\right) \nabla^{2} w^{(2)}=0 \quad \text { in } z>0,
$$

for which the boundary conditions at the disturbed interface are written, in terms of $w^{(1)}$ and $w^{(2)}$, as

$$
\begin{gathered}
\frac{\partial w^{(1)}}{\partial z}=\frac{\partial w^{(2)}}{\partial z}, \quad \frac{\partial h}{\partial t}=w^{(1)}=w^{(2)} \\
\mu_{1}\left(\Delta-\frac{\partial^{2}}{\partial z^{2}}\right) w^{(1)}=\hat{\alpha}\left(\Delta-\frac{\partial^{2}}{\partial z^{2}}\right) w^{(2)} \\
-\left(\rho_{2} \frac{\partial^{2} w^{(2)}}{\partial t \partial z}-\hat{\alpha} \nabla^{2} \frac{\partial w^{(2)}}{\partial z}\right)+2 \hat{\alpha} \Delta \frac{\partial w^{(2)}}{\partial z}+\left(\rho_{1} \frac{\partial^{2} w^{(1)}}{\partial t \partial z}-\mu_{1} \nabla^{2} \frac{\partial w^{(1)}}{\partial z}\right)-2 \mu_{1} \Delta \frac{\partial w^{(1)}}{\partial z} \\
+\left(\rho_{2}-\rho_{1}\right) a \Delta h+\gamma \Delta^{2} h=0
\end{gathered}
$$

and the conditions away from the interface are

$$
w^{(1)} \rightarrow 0 \text { as } z \rightarrow-\infty, \quad w^{(2)} \rightarrow 0 \quad \text { as } z \rightarrow \infty
$$

The solutions to Eqs(3.3.9) to satisfy (3.3.10e,f) are expressed as

$$
w^{(1)}=A^{(1)} \exp (k z)+B^{(1)} \exp \left(q_{1} z\right), \quad w^{(2)}=A^{(2)} \exp (-k z)+B^{(2)} \exp \left(-q_{2} z\right),
$$

with $q_{1}$ and $q_{2}$, defined by

$$
q_{1}=\sqrt{k^{2}+\frac{n \rho_{1}}{\mu_{1}}}, \quad q_{2}=\sqrt{k^{2}+\frac{n \rho_{2}}{\hat{\alpha}}} .
$$

After substituting (3.3.11) into the boundary conditions (3.3.10a-d), we get an inhomogeneous system of linear equations for $A^{(1)}, B^{(1)}, A^{(2)}$ and $B^{(2)}$ which is solvable if and only if the determinant of the coefficient matrix vanishes. After a straightforward but tedious analysis we have the dispersion relation:

$$
\begin{gathered}
-\left[1+\frac{1}{n^{2}}\left(\left(\alpha_{1}-\alpha_{2}\right) a k+\frac{\gamma k^{3}}{\rho_{1}+\rho_{2}}\right)\right]\left(\alpha_{2} q_{1}+\alpha_{1} q_{2}-k\right)-4 k \alpha_{1} \alpha_{2} \\
+4 \frac{k^{2}}{n} \frac{\mu_{1}-\hat{\alpha}}{\rho_{1}+\rho_{2}}\left[\alpha_{2} q_{1}-\alpha_{1} q_{2}+\left(\alpha_{1}-\alpha_{2}\right) k\right]+4 \frac{k^{3}}{n^{2}}\left(\frac{\mu_{1}-\hat{\alpha}}{\rho_{1}+\rho_{2}}\right)^{2}\left(q_{1}-k\right)\left(q_{2}-k\right)=0,
\end{gathered}
$$

where

$$
\alpha_{1}=\frac{\rho_{1}}{\rho_{1}+\rho_{2}}, \quad \alpha_{2}=\frac{\rho_{2}}{\rho_{1}+\rho_{2}} .
$$




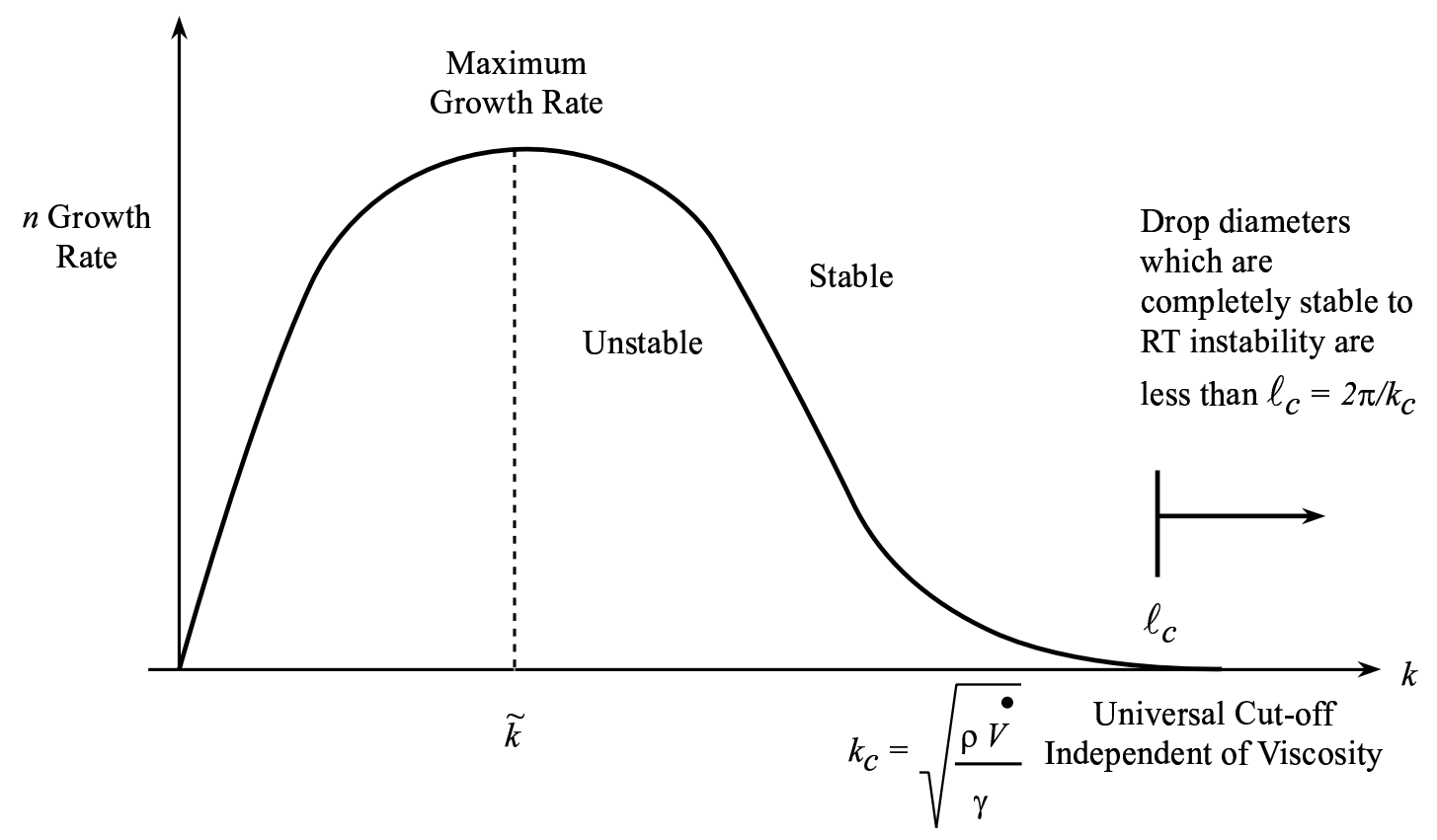

Figure 6: Schematic of a dispersion relation for Rayleigh-Taylor instability. The curve depends strongly on viscosity, relaxation and retardation times as shown in figures 7,8 and $9 . \tilde{k}$ is the wave number of the most dangerous wave.

Then the experiment shows $\rho_{2} \gg \rho_{1}$, for which $\alpha_{2} \rightarrow 1$ and $\alpha_{1} \rightarrow 0$. Moreover $\mu_{1} \ll \hat{\alpha}$ in the experiment, so that (3.3.13) reduces to

$$
-\left[1+\frac{1}{n^{2}}\left(-a k+\frac{\gamma k^{3}}{\rho_{2}}\right)\right]-4 \frac{k^{2}}{n} \frac{\hat{\alpha}}{\rho_{2}}+4 \frac{k^{3}}{n^{2}}\left(\frac{\hat{\alpha}}{\rho_{2}}\right)^{2}\left(q_{2}-k\right)=0 .
$$

The equation (3.3.15) approximates (3.3.13) with only a small error; it is appropriate for RayleighTaylor instability in a vacuum.

The solution of (3.3.13) gives rise to a dispersion relation of the type shown in figure 6. The border of stability is given by a critical wavenumber with stability only when

$$
k>k_{c}=\sqrt{\frac{\rho \dot{V}}{\gamma}}
$$

independent of viscosity, relaxation or retardation time. Dispersion relations for our experiments are presented in figures 7-9 (beginning on page 19). 


\subsection{Viscoelastic Potential Flow Analysis of Stability}

The theory of viscoelastic potential flows has been considered by Joseph and Liao [1994]. They examined the conditions under which potential flows satisfy the equations governing viscoelastic fluids. In viscoelastic potential flows the velocity is given as the gradient of a potential $u=\nabla \phi$ and $\nabla^{2} \phi=0$; this is an enormous simplification of analysis. Of course, the continuity of the velocity and shear stress at the interface must be sacrificed; the stress in a viscoelastic potential flow is evaluated on $\boldsymbol{u}=\nabla \phi$ and is, in general, not zero. The failure of potential flows to satisfy no slip conditions is fatal in problems with solid surfaces and boundary layer analysis must be used. However, the boundary layers at air-liquid surfaces resolve a discontinuity in the gradient of velocity rather than the velocity and the effects of these layers get smaller and smaller as the Reynolds numbers get larger. In any case it is never necessary or useful to consider inviscid fluids when invoking potential flow.

Rayleigh-Taylor instability at an air-liquid or vacuum-liquid surface is one of the many cases in which accurate results may be obtained using potential flow. For viscous potential flow the only place where the viscosity enters is in the normal component of the viscous stress. The dispersion relations for viscous flow and viscous potential flow derived in JBB, though different, give values for the wave number and the growth rate of the most dangerous wave that are in good agreement. Viscous potential theory yields values for the wave number that are about 2 percent higher, and values for the growth rate that are about 8.8 percent higher, than the corresponding values from fully viscous theory (JBB, table 3 ). This shows that the main physical effect of viscosity is on the normal stress balance.

The results given in JBB carry over to viscoelastic potential flows as we now show. We now require for each fluid that the potential $\phi$ gives the velocity disturbance $(\boldsymbol{u}=\nabla \phi)$ and satisfies the Laplace equation

$$
\nabla^{2} \phi=0,
$$

and the pressure disturbance is given by Bernoulli's equation

$$
\rho \frac{\partial \phi}{\partial t}+p+\rho a z=-\frac{\rho}{2}|\nabla \phi|^{2} \approx 0,
$$

against the same undisturbed state that was given in 3.3. Then the boundary conditions are given by $(3.3 .5 \mathrm{~b}, \mathrm{e})$ at the disturbed interface and $(3.3 .10 \mathrm{e}, \mathrm{f})$ away from the interface. The normal stress 
balance (3.3.5e) is now written, using (3.4.2), as

$$
\rho_{2} \frac{\partial \phi^{(2)}}{\partial t}+\tau_{33}^{(2)}+\rho_{2} a h-\left(\rho_{1} \frac{\partial \phi^{(1)}}{\partial t}+\tau_{33}^{(1)}+\rho_{1} a h\right)=-\gamma \Delta h
$$

where

$$
\frac{\tau_{33}}{2 \mu}=e_{33}=\frac{\partial w}{\partial z}=\frac{\partial^{2} \phi}{\partial z^{2}} .
$$

Thus the solutions to (3.4.1) that vanish respectively as $z \rightarrow \pm \infty$ may be expressed as

$$
w^{(1)}=A^{(1)} \exp (k z) \text { in } z<0, \quad w^{(2)}=A^{(2)} \exp (-k z) \text { in } z>0 .
$$

Substitution of these into the boundary conditions using (3.3.10b) leads to the dispersion relation:

$$
1=\frac{\alpha_{2}-\alpha_{1}}{n^{2}} k a-\frac{k^{3} \gamma}{n^{2}\left(\rho_{2}+\rho_{1}\right)}-\frac{2 k^{2}}{n} \frac{\hat{\alpha}+\mu_{1}}{\rho_{2}+\rho_{1}} .
$$

Without much loss of generality, we may put $\alpha_{1}=0, \alpha_{2}=1$ and $\hat{\alpha} \gg \mu_{1}$, so that the dispersion relation becomes

$$
1=\frac{k a}{n^{2}}-\frac{k^{3} \gamma}{n^{2} \rho_{2}}-\frac{2 k^{2}}{n} \frac{\hat{\alpha}}{\rho_{2}}
$$

which can then be written as a cubic equation for the growth rate $n$.

It is interesting to note here that (3.4.7) for viscoelastic potential flow gives the same growth rate that is given from the dispersion relation (3.3.15) for fully viscous flow if $q_{2}$ involved in (3.3.15) is approximated as

$$
q_{2}-k=\sqrt{k^{2}+\frac{n \rho_{2}}{\hat{\alpha}}}-k \approx \frac{n \rho_{2}}{2 k \hat{\alpha}}
$$

i.e., under the condition that

$$
\frac{n \rho_{2}}{2 k \hat{\alpha}} \ll 1
$$

Thus, under this condition, the theory of viscoelastic potential flow may provide a good approximation of the fully viscous theory.

\subsection{Comparison of the Exact and Potential Flow Analysis}

Based on the data for the experimental conditions cited in tables 1 and 2, the dispersion relation (3.3.15) is used to calculate the stability conditions, and the results are depicted in figure 7: $2 \% \mathrm{PO}$ $\left(M_{s}=2\right)$; figure 8: $2 \% \mathrm{PO}\left(M_{s}=3\right)$; and figure 9: $2 \% \mathrm{PAA}\left(M_{s}=3\right)$. In each of the figures several 
plots of the dispersion relation (3.3.15) are shown for a fixed (known) value of the relaxation time and various assumed values of the retardation time $\lambda_{2}$. The growth rates are computed at increments in the wavenumber of $200 \mathrm{~m}^{-1}$ from $k=0$ to the critical value. The dispersion relation (3.4.7) from viscoelastic potential theory gives rise to graphs that are nearly identical to those in figures 7-9.

For comparison of (3.4.7) and (3.3.15), values of the wavenumber $k$, wavelength $\ell$ and growth rate $n$ of the most dangerous wave are shown in table 3,2\% PO $\left(M_{s}=2\right)$; table 4, 2\% PO $\left(M_{s}=3\right)$; and table 5, 2\%PAA $\left(M_{s}=3\right)$. These results show that the set of values of the growth rate and the wavenumber given by the viscoelastic potential analysis and the corresponding set of values obtained from the exact stability analysis are at the same level of good agreement as in the Newtonian case. The wave number predicted from viscoelastic potential analysis is greater than the corresponding value from fully viscoelastic theory by between 0 and $5.4 \%$ (with two exceptions); the growth rates from viscoelastic potential analysis are between $8.5 \%$ and $9.0 \%$ higher than predicted by fully viscoelastic theory, except at the smallest values of $\lambda_{2}$.

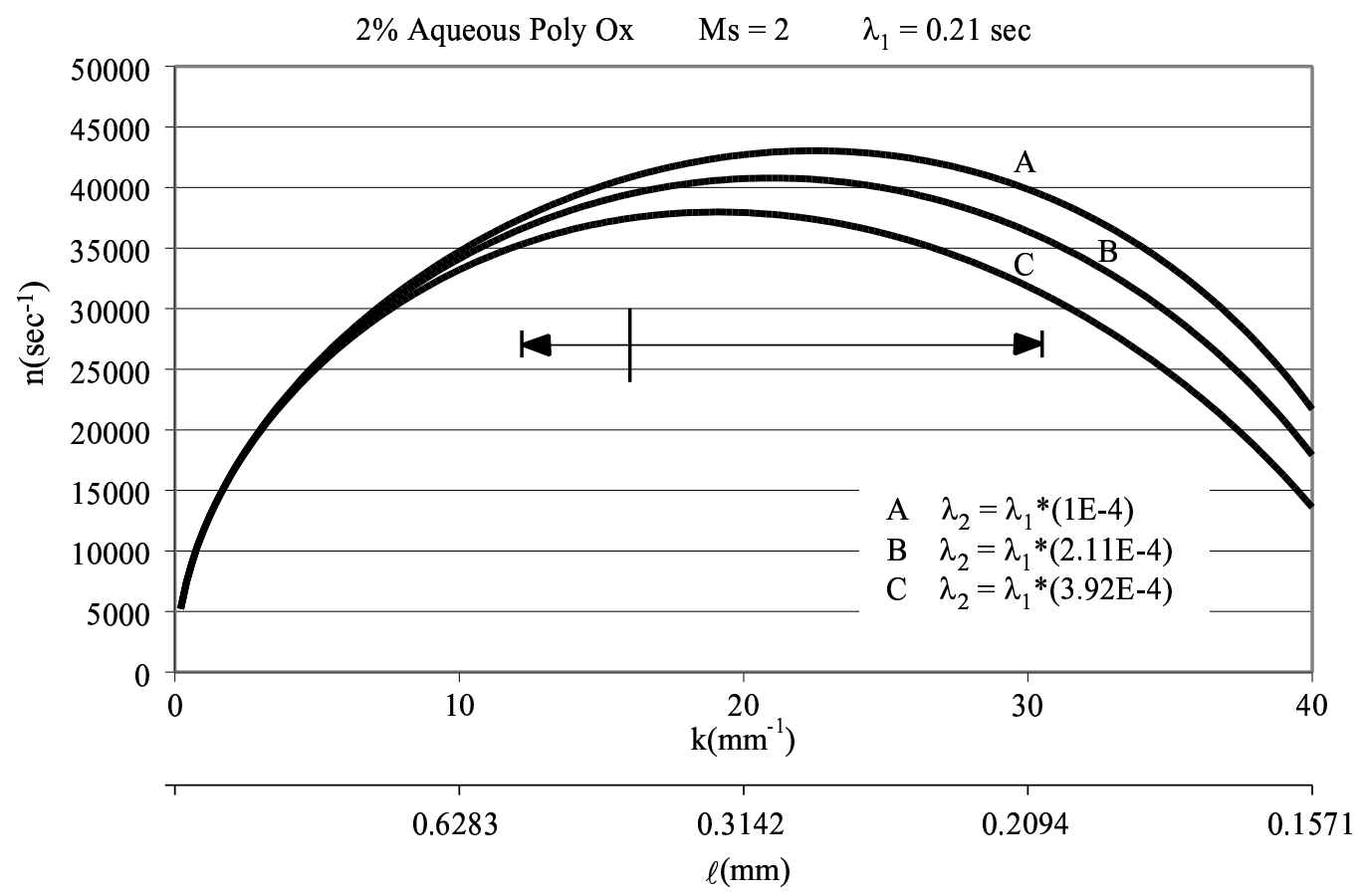

Figure 7: The growth rate $\mathrm{n}$ versus the wave number $\mathrm{k}$ from (3.3.15) for $2 \% \mathrm{PO}\left(M_{s}=2\right)$. The average wavelength and scatter from a very early time in the experiment are indicated. 


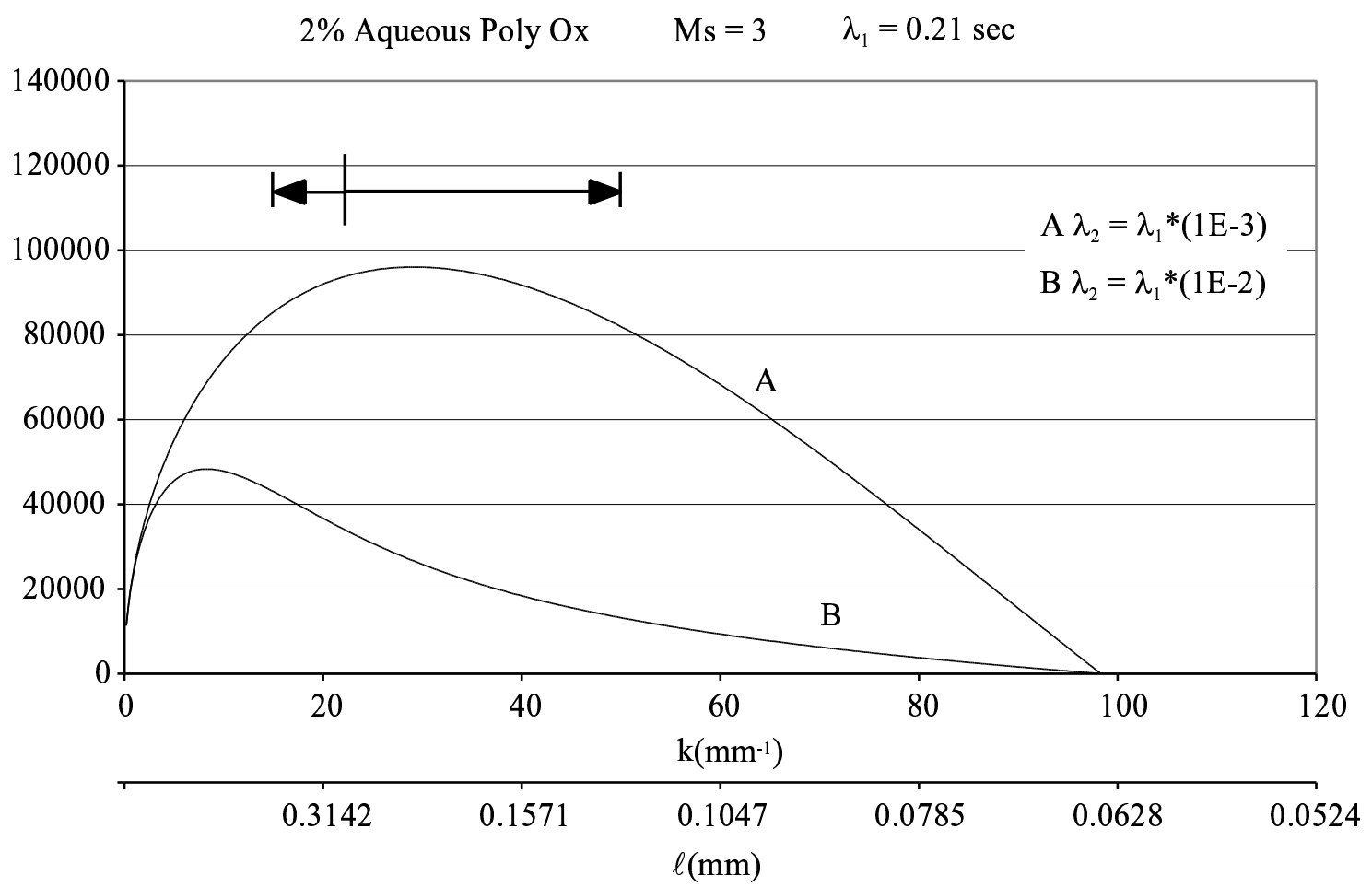

Figure 8: The growth rate $\mathrm{n}$ versus the wave number $\mathrm{k}$ from (3.3.15) for $2 \% \mathrm{PO}\left(M_{S}=3\right)$. The average wavelength and scatter from a very early time in the experiment are indicated. 


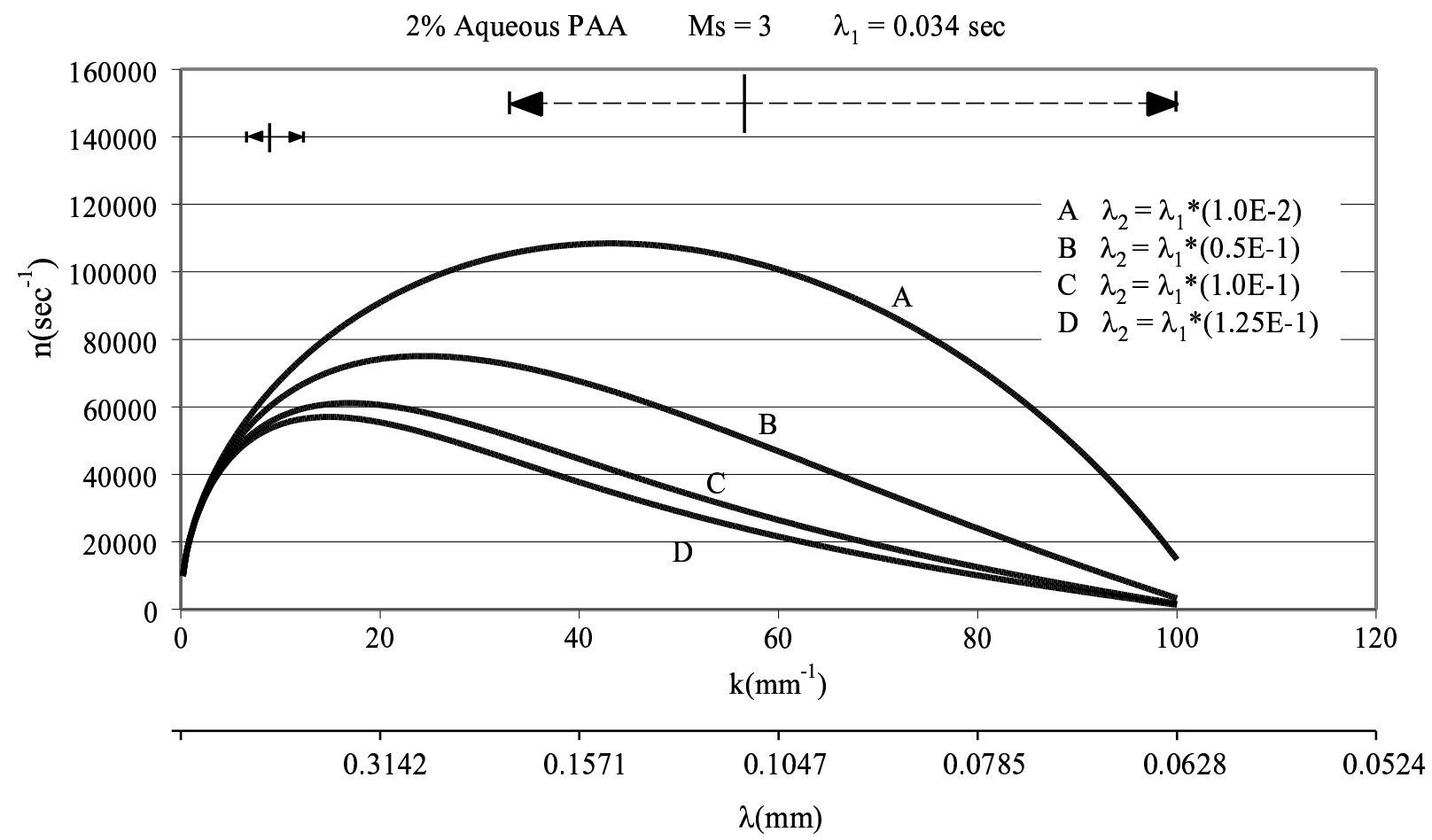

Figure 9: The growth rate $\mathrm{n} v \mathrm{~s}$ the wavenumber $\mathrm{k}$ from (3.3.15) for $2 \%$ PAA $\left(M_{S}=3\right)$. The average wavelength and scatter from a very early time in the experiment are indicated. Also shown by dotted lines are the average wavelength and scatter for the set of waves of small wavelength which appear to be superimposed on the long wavelength waves. 


\begin{tabular}{|c||r|r|r||r|r|r|r|r|}
\hline \multicolumn{1}{|c||}{} & \multicolumn{3}{c||}{ Fully Viscoelastic } & \multicolumn{2}{c|}{ Viscoelastic potential } & \multicolumn{2}{c|}{ Percent difference } \\
\cline { 2 - 9 }$\lambda_{2}[1 / \mathrm{sec}]$ & $k\left[\mathrm{~m}^{-1}\right]$ & $\ell[\mathrm{mm}]$ & $n[1 / \mathrm{sec}]$ & $k\left[\mathrm{~m}^{-1}\right]$ & $\ell[\mathrm{mm}]$ & $n[1 / \mathrm{sec}]$ & $k$ & $n$ \\
\hline$\lambda_{1} / 5$ & 600 & 10.472 & 6331.7 & 800 & 7.8539 & 6870.9 & 33.3 & 8.5 \\
\hline$\lambda_{1} / 8$ & 1000 & 6.2832 & 7425.1 & 1000 & 6.2832 & 8077.7 & 0 & 8.8 \\
\hline$\lambda_{1} / 10$ & 1000 & 6.2832 & 7991.5 & 1200 & 5.2359 & 8684.8 & 20.0 & 8.2 \\
\hline$\lambda_{1} / 20$ & 1800 & 3.4907 & 10061.5 & 1800 & 3.4907 & 10945.9 & 0 & 8.8 \\
\hline$\lambda_{1} / 100$ & 4800 & 1.3090 & 17000.0 & 5000 & 1.2566 & 18489.8 & 4.2 & 8.8 \\
\hline$\lambda_{1} / 1000$ & 15000 & 0.4189 & 32238.7 & 15800 & 0.3977 & 34849.6 & 5.3 & 8.1 \\
\hline$\lambda_{1} / 10000$ & 22400 & 0.2805 & 43036.2 & 23600 & 0.2662 & 45074.9 & 5.4 & 4.7 \\
\hline 0 & 24200 & 0.2596 & 45697.3 & 25000 & 0.2513 & 47119.7 & 3.3 & 3.1 \\
\hline
\end{tabular}

Table 3: $2 \%$ PO $\left(M_{s}=2\right)$. Values of the wavenumber $\mathrm{k}$, wavelength $\ell$ and growth rate $\mathrm{n}$ of the most dangerous wave for the experimental conditions given in tables 1 and 2; the retardation time $\lambda_{2}$ is changed against the relaxation time $\lambda_{1}$. The values of $\mathrm{k}$ and $\mathrm{n}$ predicted by viscoelastic potential theory are higher than the correpsonding fully viscoelastic predictions. The differences are indicated as a percentage of the fully viscoelastic values. 


\begin{tabular}{|c||r|r|r||r|r|r|r|r|}
\hline \multicolumn{1}{|c||}{} & \multicolumn{3}{c||}{ Fully Viscoelastic } & \multicolumn{2}{c|}{ Viscoelastic potential } & \multicolumn{2}{c|}{ Percent difference } \\
\cline { 2 - 9 }$\lambda_{2}[1 / \mathrm{sec}]$ & $k\left[\mathrm{~m}^{-1}\right]$ & $\ell[\mathrm{mm}]$ & $n[1 / \mathrm{sec}]$ & $k\left[\mathrm{~m}^{-1}\right]$ & $\ell[\mathrm{mm}]$ & $n[1 / \mathrm{sec}]$ & $k$ & $n$ \\
\hline$\lambda_{1} / 5$ & 1200 & 5.2359 & 17925.3 & 1200 & 5.2359 & 19496.0 & 0 & 8.8 \\
\hline$\lambda_{1} / 8$ & 1600 & 3.9269 & 20968.4 & 1600 & 3.9269 & 22801.0 & 0 & 8.7 \\
\hline$\lambda_{1} / 10$ & 1800 & 3.4907 & 22584.4 & 1800 & 3.4907 & 24549.0 & 0 & 8.7 \\
\hline$\lambda_{1} / 20$ & 3000 & 2.0944 & 28424.1 & 3000 & 2.0944 & 30915.1 & 0 & 8.8 \\
\hline$\lambda_{1} / 100$ & 8200 & 0.7662 & 48320.5 & 8400 & 0.7480 & 52541.6 & 2.4 & 8.7 \\
\hline$\lambda_{1} / 1000$ & 29200 & 0.2152 & 96037.0 & 30600 & 0.2053 & 103960 & 4.8 & 8.2 \\
\hline$\lambda_{1} / 10000$ & 49400 & 0.1272 & 138925 & 51600 & 0.1218 & 145138 & 4.5 & 4.4 \\
\hline 0 & 55600 & 0.1130 & 152570 & 56600 & 0.1110 & 155111 & 1.8 & 1.7 \\
\hline
\end{tabular}

Table 4: $2 \%$ PO $\left(M_{s}=3\right)$. Values of the wavenumber $\mathrm{k}$, wavelength $\ell$ and growth rate $\mathrm{n}$ of the most dangerous wave for the experimental conditions given in tables 1 and 2; the retardation time $\lambda_{2}$ is changed against the relaxation time $\lambda_{1}$. The values of $\mathrm{k}$ and $\mathrm{n}$ predicted by viscoelastic potential theory are higher than the correpsonding fully viscoelastic predictions. The differences are indicated as a percentage of the fully viscoelastic values. 


\begin{tabular}{|c||r|c|c||r|r|r|r|r|}
\hline \multicolumn{1}{|c||}{} & \multicolumn{3}{c||}{ Fully Viscoelastic } & \multicolumn{2}{c|}{ Viscoelastic potential } & \multicolumn{2}{c|}{ Percent difference } \\
\cline { 2 - 9 }$\lambda_{2}[1 / \mathrm{sec}]$ & $k\left[\mathrm{~m}^{-1}\right]$ & $\ell[\mathrm{mm}]$ & $n[1 / \mathrm{sec}]$ & $k\left[\mathrm{~m}^{-1}\right]$ & $\ell[\mathrm{mm}]$ & $n[1 / \mathrm{sec}]$ & $k$ & $n$ \\
\hline$\lambda_{1} / 5$ & 11200 & 0.5610 & 49081.4 & 11400 & 0.5512 & 53350.0 & 1.8 & 8.7 \\
\hline$\lambda_{1} / 8$ & 14800 & 0.4245 & 57009.4 & 15200 & 0.4134 & 61948.0 & 2.7 & 8.7 \\
\hline$\lambda_{1} / 10$ & 17000 & 0.3696 & 61112.1 & 17400 & 0.3611 & 66389.7 & 2.4 & 8.6 \\
\hline$\lambda_{1} / 20$ & 24400 & 0.2575 & 75051.4 & 25400 & 0.2474 & 81393.6 & 4.1 & 8.5 \\
\hline$\lambda_{1} / 100$ & 43200 & 0.1454 & 108441 & 45400 & 0.1384 & 115536 & 5.1 & 6.5 \\
\hline$\lambda_{1} / 1000$ & 56400 & 0.1114 & 133671 & 57800 & 0.1087 & 136333 & 2.5 & 2.0 \\
\hline$\lambda_{1} / 10000$ & 59000 & 0.1065 & 138403 & 59400 & 0.1058 & 139290 & 0.7 & 0.6 \\
\hline 0 & 59400 & 0.1058 & 139007 & 59800 & 0.1051 & 139633 & 0.7 & 0.5 \\
\hline
\end{tabular}

Table 5: 2\% PAA $\left(M_{s}=3\right)$. Values of the wavenumber $\mathrm{k}$, wavelength $\ell$ and growth rate $\mathrm{n}$ of the most dangerous wave for the experimental conditions given in tables 1 and 2; the retardation time $\lambda_{2}$ is changed against the relaxation time $\lambda_{1}$. The values of $\mathrm{k}$ and $\mathrm{n}$ predicted by viscoelastic potential theory are higher than the correpsonding fully viscoelastic predictions. The differences are indicated as a percentage of the fully viscoelastic values. 


\subsection{The effect of acceleration on instability}

The value of the acceleration $\dot{V}$ is a major factor in Rayleigh-Taylor instability. The wavelength of the most rapidly growing disturbance decreases and the growth rate increases strongly as the acceleration $\dot{V}$ is increased (figure 8). The discussion of acceleration given in section 3.2 suggests that the force producing acceleration at early times is the pressure difference across the drop measured basically by the dynamic pressure. The breakup of a drop even at high speed may be very retarded or even suppressed when the air density is small, as at high altitudes. The analysis suggests also that the acceleration does not depend strongly on the rheology of the drop even though rheology affects the growth rate strongly.

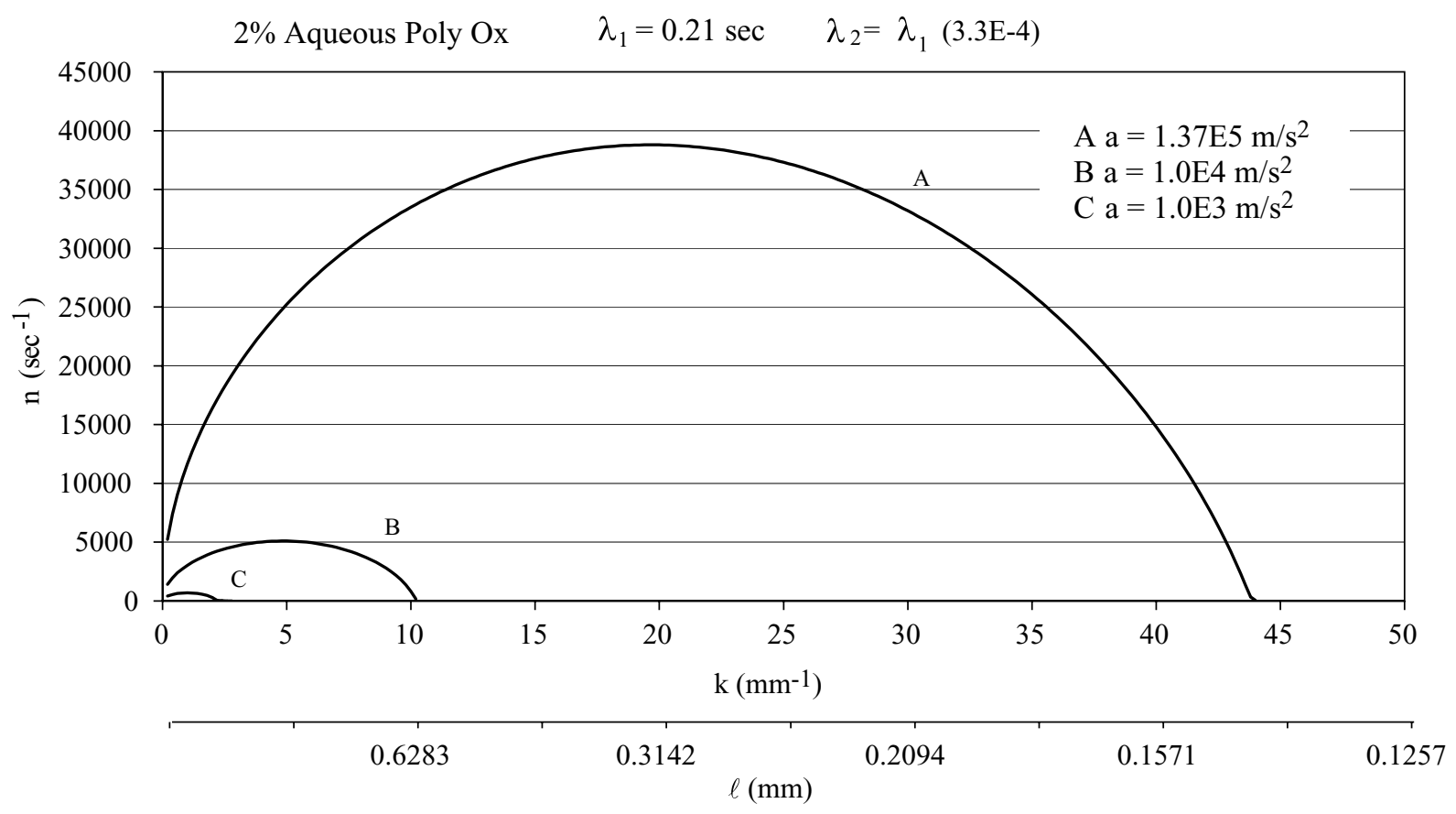

Figure 10. The effect of acceleration on the dispersion relation for $2 \%$ PO.

\section{Comparison of theory and experiment}

We now compare the Rayleigh-Taylor stability theory with experiments on drop breakup for the three viscoelastic cases discussed in section 2. For comparison, we repeat results from JBB for a $1.0 \mathrm{~kg} / \mathrm{m} . \mathrm{sec}$ silicone oil whose viscosity nearly matches the $0.96 \mathrm{~kg} / \mathrm{m} . \mathrm{sec}$ polyacrylamide. 
Figure 11, taken from JBB, shows the waves on drops of this Newtonian liquid at very early times in the motion at shock Mach numbers of 2 and 3.

Silicone Oil 1000

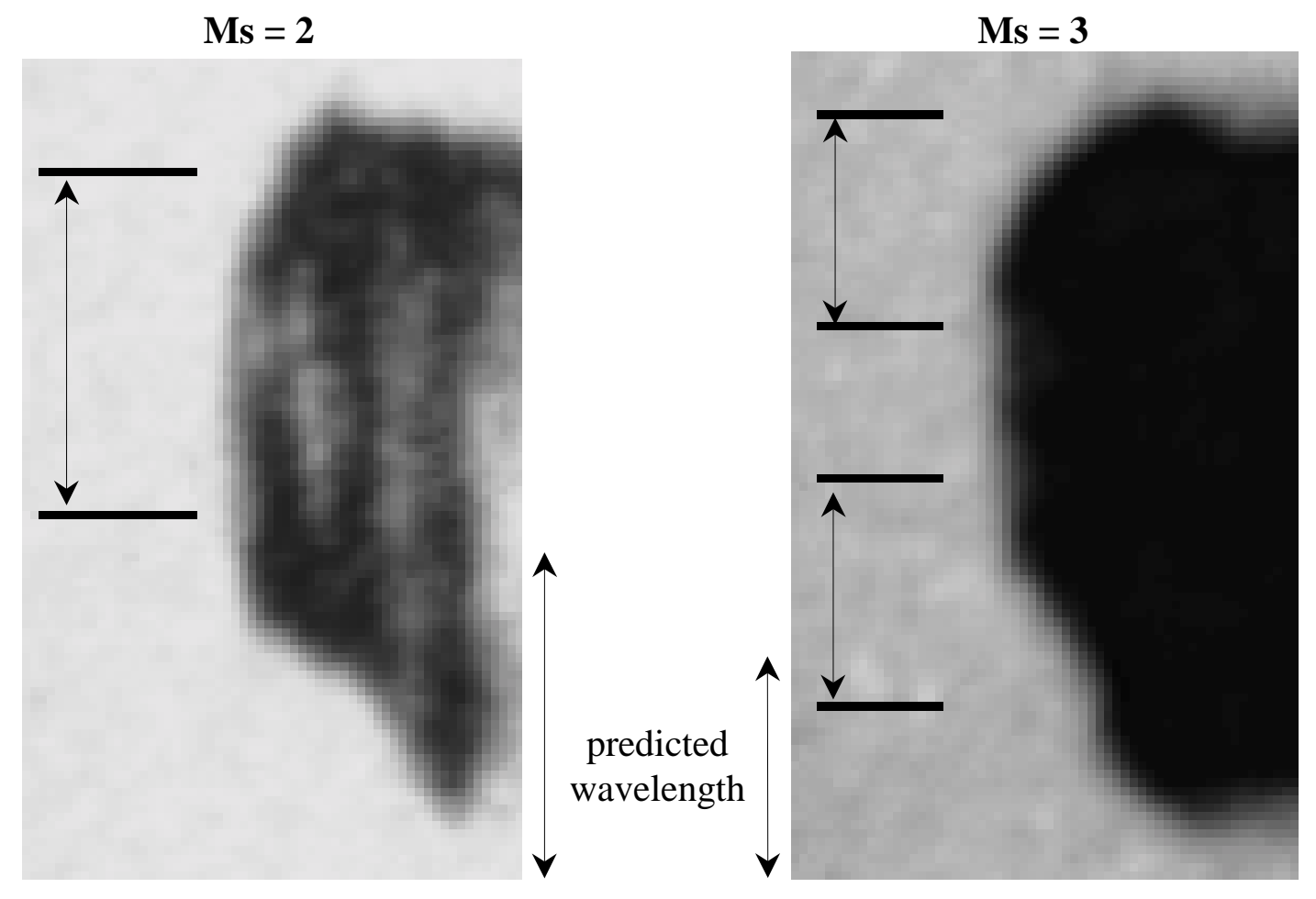

Figure 11. Rayleigh-Taylor waves in silicone oil (1 kg/m.sec). (From Joseph, Belanger, and Beavers 1999. )

The waves on both the polyox and polyacrylamide were smaller and more difficult to identify than the waves on the Newtonian liquids presented in JBB. For example, the measured average wavelengths for the $1.0 \mathrm{~kg} / \mathrm{m} . \mathrm{sec}$ silicone oil (Figure 11) are about $2.0 \mathrm{~mm}$ and $1.25 \mathrm{~mm}$ for shock Mach numbers of 2 and 3 respectively, while the corresponding values for the $2 \%$ polyox solution are $0.39 \mathrm{~mm}$ and $0.20 \mathrm{~mm}$. In an attempt to identify the waves more clearly on the computer screen Adobe Photoshop ${ }^{\mathrm{TM}}$ was used to exaggerate the contrast. We then measured the lengths of the waves by first locating the troughs across the front of the drop on the computer screen, and then measuring the distance between troughs in pixels which were finally converted to millimeters using a predetermined scaling factor for each frame. The enhanced contrast images are shown in figure 12 for the $2 \%$ aqueous polyox and figure 13 for the $2 \%$ polyacrylamide. The tick marks identify the wave troughs. Like the Newtonian liquids in JBB, the troughs are easier to 


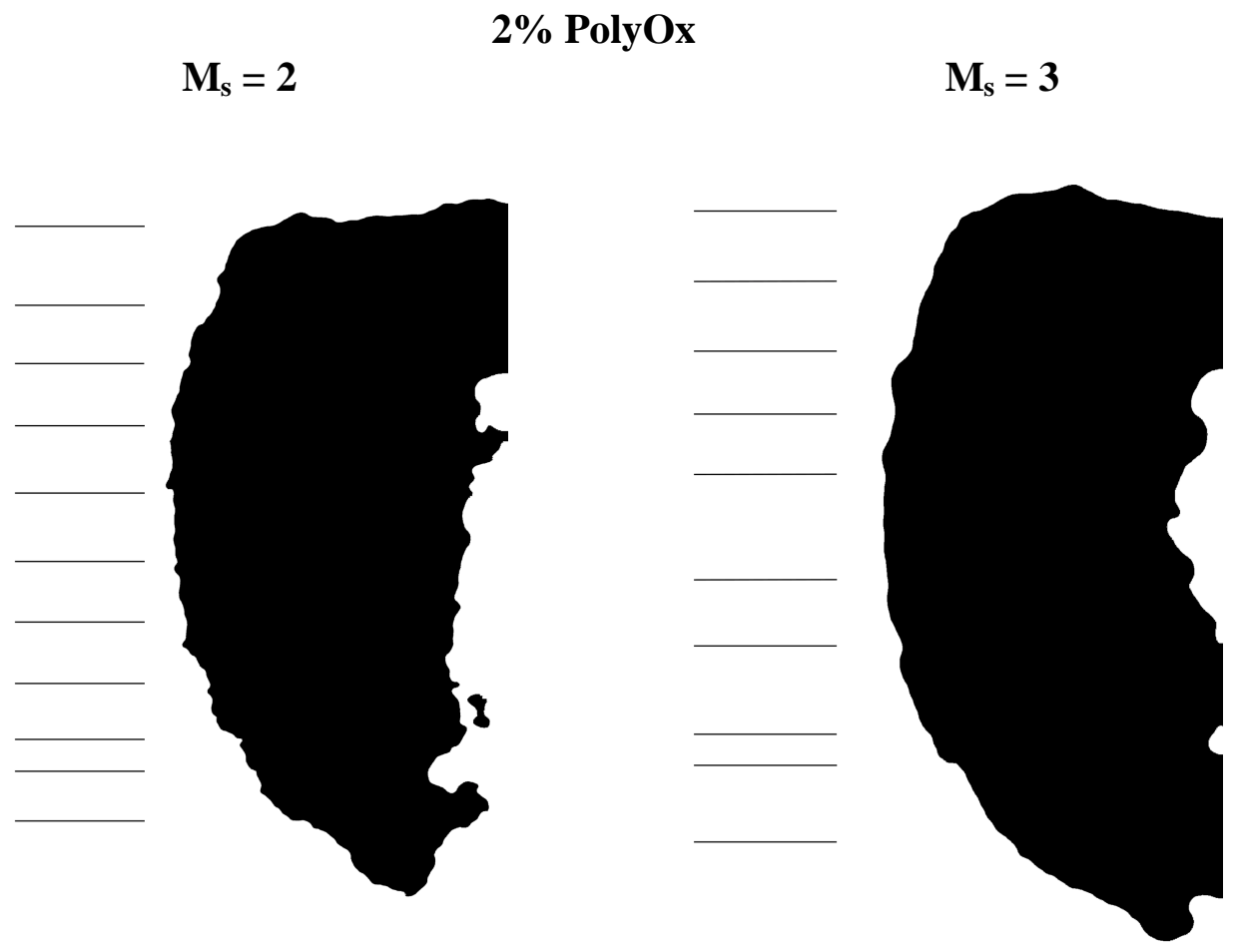

Figure 12. Rayleigh-Taylor waves in $2 \%$ aqueous polyox.

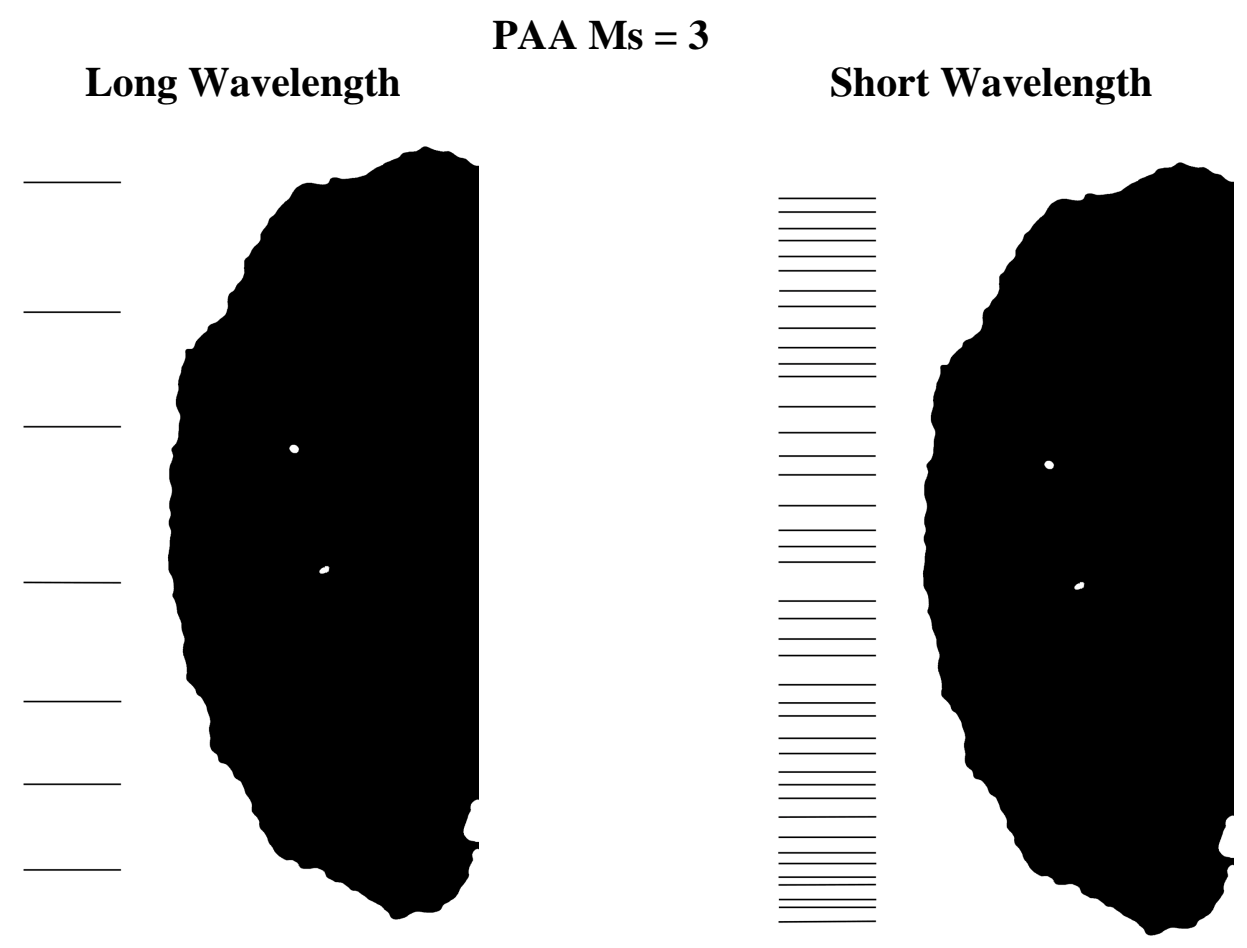

Figure 13. Rayleigh-Taylor waves in $2 \%$ aqueous polyocrylamide. 
identify on the computer screen than in a printed figure. The length of the waves increases with time because the waves are ultimately forced apart by high pressures in the wave troughs; from this it follows that the length of unstable waves should be measured at the earliest times.

Polyacrylamide

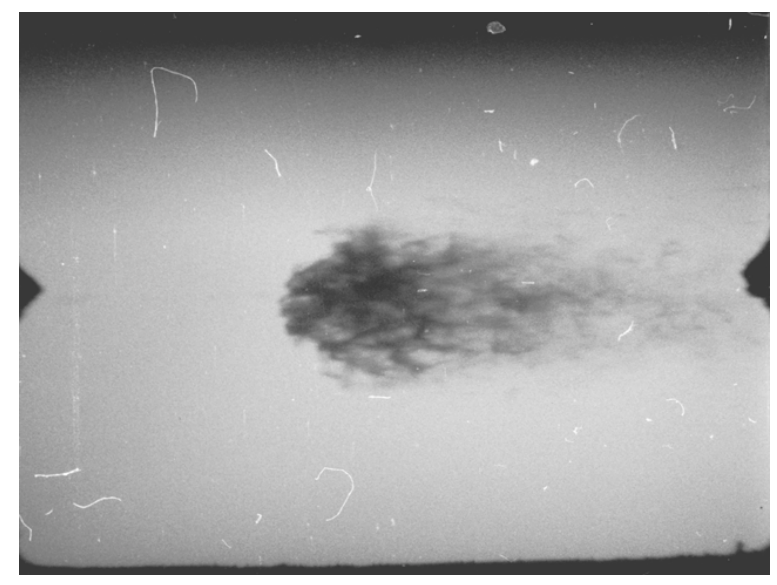

$2 \%$ PolyOx

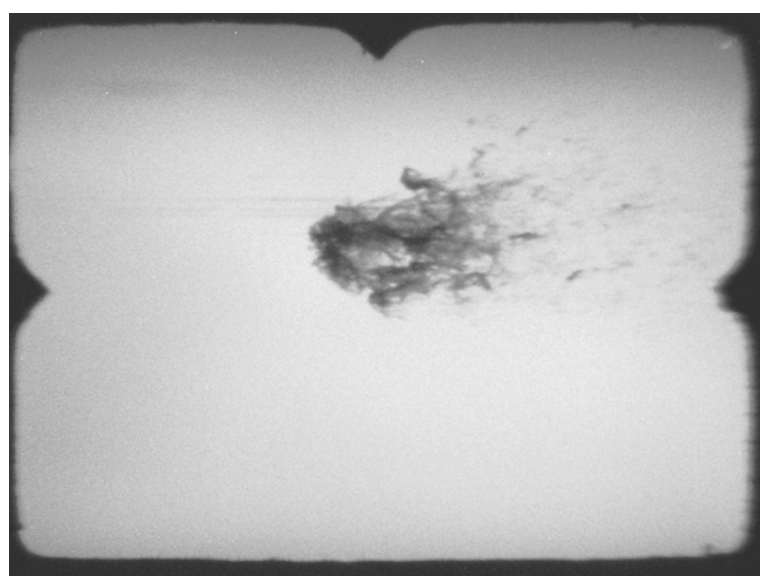

Silicone Oil

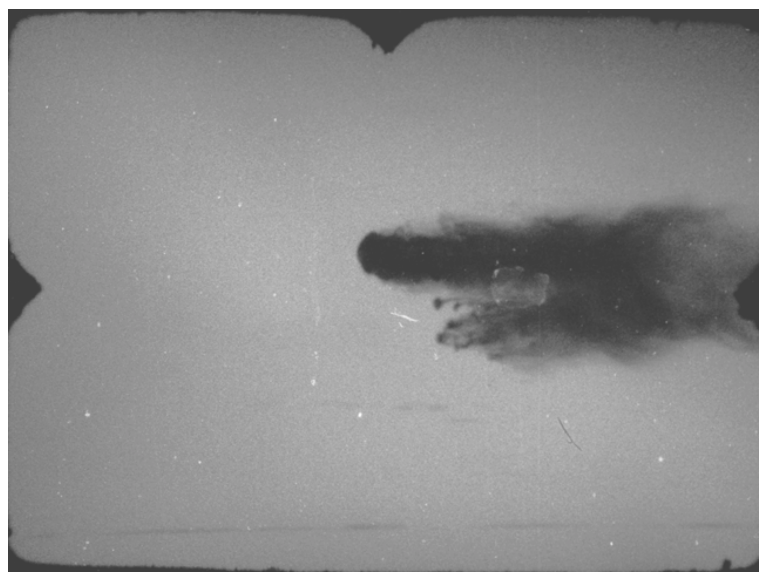

Silicone Oil

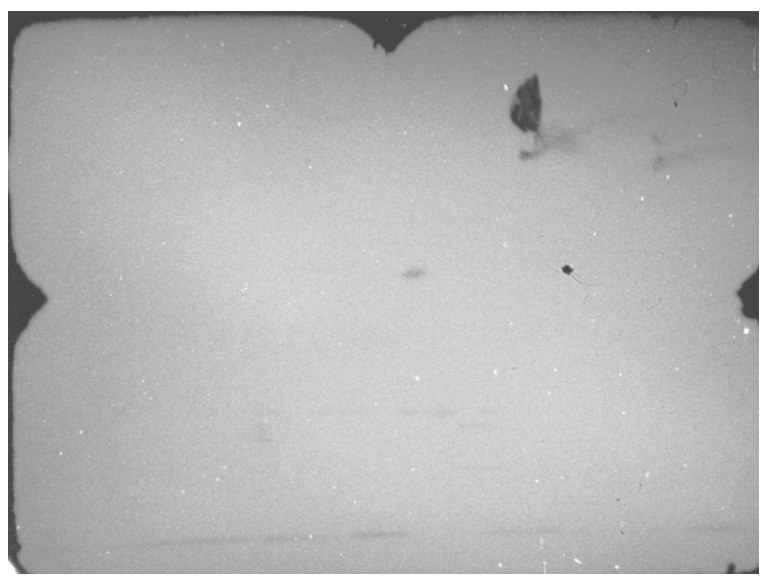

Figure 14. Droplet configurations for $2 \%$ PAA, $2 \%$ PO, and two different silicone oils at 170 $\mu$ secs after passing of the shock over the drop under the same conditions $\left(M_{s}=3\right)$. The top pair of photographs compares PAA with a silicone oil of approximately the same viscosity $(1 \mathrm{~kg} / \mathrm{m} . \mathrm{sec})$. The bottom pair compares $2 \%$ PO (viscosity $=35 \mathrm{~kg} / \mathrm{m} . \mathrm{sec})$ with a silicone oil that has a viscosity of about one-third that of the PO $(10 \mathrm{~kg} / \mathrm{m} . \mathrm{sec})$.

There is some uncertainty in the measurements of the wavelengths from the 2\% PAA picture because there appeared to be two sets of waves, a distinct set of waves with an average wavelength of $0.70 \mathrm{~mm}$ with a second set of smaller waves superimposed on the larger waves. The wavelengths of the smaller waves were very irregular, with values between approximately 
$0.05 \mathrm{~mm}$ and $0.24 \mathrm{~mm}$. Smaller, but less distinct, waves could also be identified over parts of the front face of the polyox drops.

On figures 7-9 we graph dispersion relations corresponding to measured data given in tables 1 and 2 . The retardation time $\lambda_{2}$ is a fitting parameter. The dispersion graphs are sensitive to values of $\lambda_{2}$ as is shown in figures $7-9$, where for each figure values for $\lambda_{2}$ have been chosen to yield curves such that the wavelengths of maximum growth are close to the interval of instability defined from the experiments, which is also included on the figures. From these we may estimate a $\lambda_{2}$ which centers the wavelength of maximum growth in the interval of instability. The estimated values of $\lambda_{2}$ needed to achieve agreement are uniformly small ranging from $\lambda_{2} \approx \lambda_{1} / 5000$ for $2 \%$ aqueous polyox at $\mathrm{M}_{\mathrm{s}}=2$ to $\lambda_{2} \approx \lambda_{1} / 100$ for $2 \%$ aqueous PAA at $\mathrm{M}_{\mathrm{s}}=3$. The retardation time measuring viscous response has been interpreted as representing the effect of relaxed elastic modes (see Joseph [1990], chapter 18). Its value may change from zero, say for disturbances of duration $10^{-13} \mathrm{sec}$, to one for very long disturbances in which the viscosity is given by the area under the relaxation curve representing the decay of the shear modulus. The small value of the retardation time which matches theory and experiment reported here is just what might be expected in such an explosive and short time (10-50 $\mu \mathrm{s})$ event as produces Rayleigh-Taylor waves on drops suddenly exposed to a high speed airstream.

In the previous paragraph we have argued that $\lambda_{2}$ is not given once and for all but depends at least on some conditions of external excitation and that Rayleigh-Taylor disturbances are so fast that the response of the drops is highly elastic (small values of $\lambda_{2}$ ).

Following the approach adopted in JBB we arbitrarily defined a 'breakup' time as the time $\hat{t}_{b}$ taken for the initial amplitude $A_{o}$ of an unstable disturbance $A(t)=A_{o} e^{n t}$ to grow to $M$ times its initial value:

$$
\begin{aligned}
& M=\frac{A\left(\hat{t}_{b}\right)}{A_{o}}=e^{n t_{b}} \\
& \hat{t}_{b}=\frac{1}{n} \ln M,
\end{aligned}
$$

which implies that higher growth rates $n$ lead to faster breakup. The values for $n$ given in table 4 suggest that for a fixed shock Mach number, the viscoelastic liquids appear to start to 'breakup' 
faster than purely viscous liquids of about the same viscosity. This effect is illustrated in figure 14 which shows the configuration of drops of the test liquids at a time of $170 \mu \mathrm{secs}$ from the passing of the shock wave over the drop under the same experimental conditions $\left(M_{s}=3.0\right)$. The top two figures compare 2\% PAA with SO 1000 silicone oil (viscosities close to $1 \mathrm{~kg} / \mathrm{m} . \mathrm{sec}$ ). The PAA drop has broken up completely at this stage, whereas part of the original silicone drop can still be identified. At higher viscosities the difference in breakup is more pronounced, as shown in the lower two photographs. The $2 \%$ PO is completely broken up whereas the silicone oil remains as a distorted drop. This faster start to breakup of the viscoelastic liquids compared with the purely viscous liquid is also evident by comparison of the times listed in table 6 for liquid to begin to 'blow off' at the equator of the drop.

\begin{tabular}{|c|c|c|c|c|}
\hline \multicolumn{1}{|c|}{ Liquid } & $\begin{array}{c}\text { Shock Mach } \\
\text { No. }\end{array}$ & $\begin{array}{c}\text { Approx. } \mathbf{n} \\
(\mathbf{1} / \mathbf{s e c})\end{array}$ & $\begin{array}{c}\text { Time for } \\
\mathbf{A = 1 0} \mathbf{A}_{\mathbf{o}} \\
(\boldsymbol{\mu} \mathbf{s e c})\end{array}$ & $\begin{array}{c}\text { Experimental blow- } \\
\text { off time }(\boldsymbol{\mu} \mathbf{s e c})\end{array}$ \\
\hline SO 1000 & 3 & 48769 & 47 & 40 \\
$2 \%$ PO & 3 & 90,000 & 25 & 30 \\
$2 \%$ PO & 2 & 38,000 & 60 & 50 \\
$2 \%$ PAA & 3 & & & 35 \\
Short & & 110,000 & 20 & \\
Long & & 75,000 & 30 & \\
\hline
\end{tabular}

Table 6. Comparison of measured breakup times (defined as the time at which liquid first starts to 'blow-off' the perimeter of the drop) with predicted times calculated from equation (4.1) with $M=10$ and using values of $n$ from the fully viscoelastic analysis.

\section{Conclusions}

We compare the breakup of two viscoelastic liquids with the breakup of a purely viscous liquid of about the same viscosity under the same experimental conditions using a high-speed rotating drum camera. As described in JBB, bag-and-stamen in bag breakup occurs routinely at Weber numbers of $0\left(10^{4}-10^{5}\right)$ in the higher viscosity drops and even in viscoelastic drops showing that the presently accepted classification of breakup events, based mainly on water, does not hold generally. The early events of breakup $(<100 \mu \mathrm{s})$, flattening, and the accumulation of fluid driven away from the stagnation points, followed by fingering are universal and apply 
equally to low and high viscosity fluids and to viscoelastic fluids. At later times the drop fragments in viscoelastic fluid are much more stringy than in Newtonian fluids of comparable viscosity. This confirms that the stringiness of drop fragments persists in high speed, high Weber number flows. The movies generate time-displacement data from which accelerations of the drop may be computed in these experiments. The accelerations were $10^{4}-10^{5}$ times the acceleration of gravity, putting the drops at risk to Rayleigh-Taylor instabilities. The Rayleigh-Taylor instabilities were computed with an exact viscous theory and with a simplified theory based on viscous potential flow. It is assumed that the most dangerous wave is the one whose length gives the maximum growth rate. The simplified viscoelastic potential theory gives the critical wave length and growth rate within less than 10 percent of the exact theory. The border of instability to Rayleigh-Taylor fingers is given by a critical wave number, with stability only when

$$
k>k_{c}=\sqrt{\frac{\rho a}{\gamma}},
$$

independent of viscous and viscoelastic parameters. 


\section{Acknowledgements}

The authors acknowledge the valuable contributions of Jacques Belanger, Gerry Brenden, Abbey Eichman, and Paul Hannah.

This work was supported by U.S. Army ERDEC through Grant No. Battelle/1359051/Army, by the Army Research Office and by the National Science Foundation under grant 9622235.

\section{References}

Aitken, L.S., and Wilson, S.D.R., 1993. Rayleigh-Taylor instability in elastic liquids, J. NonNewtonian Fluid Mech., 49, 13-22.

Engel, O.G., 1958. Fragmentation of waterdrops in the zone behind an air shock, J. Res. Nat'l. Bur. Stand, 60, 245-280.

Faeth, G.M., 1996. Spray combustion phenomena, Twenty-sixth symposium on combustion, The Combustion Institute 1593-1611.

Hsiang, L-P., and Faeth, G.M., 1992. Near-limit drop deformation and secondary breakup, Int. J. Multiphase Flow, 18, 635-652.

Hwang, S.S., Liu, Z., and Reitz, R.D., 1996. Breakup mechanisms and drag coefficients of highspeed vaporizing liquid drops, Atomization and Sprays, 6, 353-376.

Joseph, D.D., 1990. Fluid Dynamics of Viscoelastic Liquids, Applied Mathematical Sciences, Vol 84, Springer-Verlag.

Joseph, D.D., Belanger, J. and Beavers, G.S. 1999. Breakup of a liquid drop suddenly exposed to a high speed airstream, Int. J Multiphase Flow, 25 (6-7) 1263-1303.

Joseph, D.D., and Liao, T.Y., 1994. Potential flows of viscous and viscoelastic fluids, J. Fluid Mech. 265, 1-23.

Joseph, D.D. and Saut, J.C., 1990. Short-wave instabilities and ill-posed initial-value problems, Theroetical Comput. Fluid Dynamics, 1, 191-227.

Kitscha, J. and Kocamustafaogullari, G., 1989. Breakup criteria for fluid particles, Int. J. Multiphase Flow, 15, 573-588.

Krzeczkowski, S.A., 1980. Measurement of liquid droplet disintegration mechanisms, Int. J. Multiphase Flow, 6, 227-239. 
Lane, W.R., 1951. Shatter of drops in stream of air. Ind. Engng Chem., 43, 1312-1317.

Liu, Y.J., 1995. Particle Motions in Non-Newtonian Fluids, Ph.D. Thesis, University of Minnesota.

Liu, Z. and Reitz, R.D., 1997. An analysis of the distortion and breakup mechanisms of highspeed liquid drops, Int. J. Multiphase Flow, 23, 631-650.

Matta, J.E. and Tytus, R.P., 1982. Viscoelastic breakup in a high velocity airstream, J. Appl. Polymer Science, 27, 397-405.

Matta, J.E., Tytus, R.P., and Harris, J., 1983. Aerodynamic atomization of polymeric solutions, Chem Eng. Commun., 19, 191-204.

Stone, H.A., 1994. Dynamics of drop deformation and breakup in viscous fluids, Annual Rev. Fluid Mech., 26, 65-102.

Taylor, G.I., 1950. The instability of liquid surfaces when accelerated in a direction perpendicular to their planes, I. Proc. Roy. Soc. A, 201, 192-196; also in The Scientific Papers of G.I. Taylor, 3 (ed. G.K. Batchelor), University Press, Cambridge (1993).

Wierzba, A., 1990. Deformation and breakup of liquid drops in a gas stream at nearly critical Weber numbers, Experiments in Fluids, 9, 59-64.

Wilcox, J.D., June, R.K., Braun, H.A. and Kelly, R.C., 1961. The retardation of drop breakup in high-velocity airstreams by polymeric modifiers. J. App. Polymer Sci., Vol V, 13, 1-6. 